\title{
Dust-Gas Scaling Relations and OH Abundance in the Galactic ISM
}

Hiep Nguyen ${ }^{1,2}$ (1) , J. R. Dawson ${ }^{1,2}$ (1), M.-A. Miville-Deschênes ${ }^{3,4}$, Ningyu Tang ${ }^{5}$ (1) Di Li $^{5,6}$ (1), Carl Heiles ${ }^{7}$, Claire E. Murray ${ }^{8}$ (1),

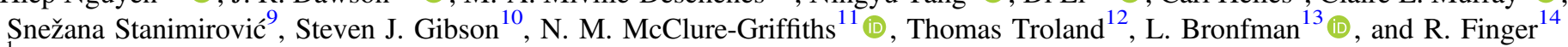

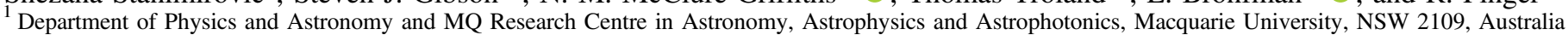
van-hiep.nguyen@hdr.mq.edu.au

${ }^{2}$ Australia Telescope National Facility, CSIRO Astronomy and Space Science, P.O. Box 76, Epping, NSW 1710, Australia

${ }^{3}$ Institut d'Astrophysique Spatiale, CNRS, Univ. Paris-Sud, Université Paris-Saclay, Bât. 121, F-91405, Orsay Cedex, France

${ }^{4}$ Laboratoire AIM, Paris-Saclay, CEA/IRFU/DAp-CNRS-Université Paris Diderot, F-91191, Gif-sur-Yvette Cedex, France

${ }^{5}$ CAS Key Laboratory of FAST, NAOC, Chinese Academy of Sciences, People's Republic of China

${ }^{6}$ University of Chinese Academy of Sciences, Beijing 100049, People's Republic of China

${ }^{7}$ Department of Astronomy, University of California, Berkeley, 601 Campbell Hall 3411, Berkeley, CA 94720-3411, USA

${ }^{8}$ Space Telescope Science Institute, 3700 San Martin Drive, Baltimore, MD 21218, USA

${ }^{9}$ Department of Astronomy, University of Wisconsin-Madison, 475 North Charter Street, Madison, WI 53706, USA

${ }^{10}$ Department of Physics and Astronomy, Western Kentucky University, Bowling Green, KY 42101, USA

${ }^{11}$ Research School of Astronomy and Astrophysics, Australian National University, Canberra, ACT 2611, Australia

${ }^{12}$ Department of Physics and Astronomy, University of Kentucky, Lexington, Kentucky 40506, USA

${ }^{13}$ Departamento de Astronomía, Universidad de Chile, Casilla 36, Santiago de Chile, Chile

14 Astronomy Department, Universidad de Chile, Camino El Observatorio 1515, 1058 Santiago, Chile

Received 2018 February 19; revised 2018 May 21; accepted 2018 May 24; published 2018 July 20

\begin{abstract}
Observations of interstellar dust are often used as a proxy for total gas column density $N_{\mathrm{H}}$. By comparing Planck thermal dust data (Release 1.2) and new dust reddening maps from Pan-STARRS 1 and 2MASS, with accurate (opacity-corrected) $\mathrm{H}$ I column densities and newly published $\mathrm{OH}$ data from the Arecibo Millennium survey and 21-SPONGE, we confirm linear correlations between dust optical depth $\tau_{353}$, reddening $E(B-V)$, and the total proton column density $N_{\mathrm{H}}$ in the range $(1-30) \times 10^{20} \mathrm{~cm}^{-2}$, along sightlines with no molecular gas detections in emission. We derive an $N_{\mathrm{H}} / E(B-V)$ ratio of $(9.4 \pm 1.6) \times 10^{21} \mathrm{~cm}^{-2} \mathrm{mag}^{-1}$ for purely atomic sightlines at $|b|>5^{\circ}$, which is $60 \%$ higher than the canonical value of Bohlin et al. We report a $\sim 40 \%$ increase in opacity $\sigma_{353}=\tau_{353} / N_{\mathrm{H}}$, when moving from the low column density $\left(N_{\mathrm{H}}<5 \times 10^{20} \mathrm{~cm}^{-2}\right)$ to the moderate column density $\left(N_{\mathrm{H}}>5 \times 10^{20} \mathrm{~cm}^{-2}\right)$ regime, and suggest that this rise is due to the evolution of dust grains in the atomic interstellar medium. Failure to account for $\mathrm{H}$ I opacity can cause an additional apparent rise in $\sigma_{353}$ of the order of a further $\sim 20 \%$. We estimate molecular hydrogen column densities $N_{\mathrm{H}_{2}}$ from our derived linear relations, and hence derive the $\mathrm{OH} / \mathrm{H}_{2}$ abundance ratio of $X_{\mathrm{OH}} \sim 1 \times 10^{-7}$ for all molecular sightlines. Our results show no evidence of systematic trends in $\mathrm{OH}$ abundance with $N_{\mathrm{H}_{2}}$ in the range $N_{\mathrm{H}_{2}} \sim(0.1-10) \times 10^{21} \mathrm{~cm}^{-2}$. This suggests that $\mathrm{OH}$ may be used as a reliable proxy for $\mathrm{H}_{2}$ in this range, which includes sightlines with both CO-dark and CO-bright gas.
\end{abstract}

Key words: dust, extinction - ISM: clouds - ISM: molecules

\section{Introduction}

Observations of neutral hydrogen in the interstellar medium (ISM) have historically been dominated by two radio spectral lines: the $21 \mathrm{~cm}$ line of atomic hydrogen ( $\mathrm{HI})$ and the microwave emission from carbon monoxide $(\mathrm{CO})$, particularly the $\operatorname{CO}(J=1-0)$ line. The former provides direct measurements of the warm neutral medium (WNM), and the cold neutral medium (CNM), which is the precursor to molecular clouds. The latter is widely used as a proxy for molecular hydrogen $\left(\mathrm{H}_{2}\right)$, often via the use of an empirical "X-factor," (e.g., Bolatto et al. 2013). The processes by which CNM and molecular clouds form from warm atomic gas sows the seeds of structure into clouds, laying the foundations for star formation. Being able to observationally track the ISM through this transition is of key importance.

However, there is strong evidence for gas not seen in either H I or CO. This undetected material is often called "dark gas," following Grenier et al. (2005). These authors found an excess of diffuse gamma-ray emission from the Local ISM, with respect to the expected flux due to cosmic-ray interactions with the gas mass estimated from H I and CO. Similar conclusions have been reached using many different tracers, including $\gamma$-rays (e.g., Abdo et al. 2010; Ackermann et al. 2012, 2011), infrared emission from dust (e.g., Blitz et al. 1990; Reach et al. 1994; Douglas \& Taylor 2007; Planck Collaboration et al. 2011, 2014a), dust extinction (e.g., Paradis et al. 2012; Lee et al. 2015), C II emission (Pineda et al. 2013; Langer et al. 2014; Tang et al. 2016), and $\mathrm{OH} 18 \mathrm{~cm}$ emission and absorption (e.g., Wannier et al. 1993; Liszt \& Lucas 1996; Barriault et al. 2010; Allen et al. 2012, 2015; Engelke \& Allen 2018).

While a minority of studies have suggested that cold, optically thick $\mathrm{H}$ I could account for almost all the missing gas mass (Fukui et al. 2015), CO-dark $\mathrm{H}_{2}$ is generally expected to be a major constituent, particularly in the envelopes of molecular clouds (e.g., Lee et al. 2015). In diffuse molecular regions, $\mathrm{H}_{2}$ is effectively self-shielded, but $\mathrm{CO}$ is typically photodissociated (Tielens \& Hollenbach 1985a, 1985b; van Dishoeck \& Black 1988; Wolfire et al. 2010; Glover \& Mac Low 2011; Lee et al. 2015; Glover \& Smith 2016), meaning that $\mathrm{CO}$ lines are a poor tracer of $\mathrm{H}_{2}$ in such environments. Indeed Herschel observations of $\mathrm{C}$ II suggest that between $20 \%-75 \%$ of the $\mathrm{H}_{2}$ in the Galactic plane may be CO-dark (Pineda et al. 2013). 
For the atomic medium, the mass of warm $\mathrm{HI}$ can be computed directly from measured line intensities under the optically thin assumption. However, cold H I with spin temperature $T_{s} \lesssim 100 \mathrm{~K}$ suffers from significant optical depth effects, leading to an underestimation of the total column density. This difficulty is generally addressed by combining H I absorption and emission profiles observed toward (and immediately adjacent to) bright, compact continuum background sources. Such studies find that the optically thin assumption underestimates the true H I column by no more than a few tens of percent along most Milky Way sightlines (e.g., Dickey et al. 1983, 2000, 2003; Heiles \& Troland 2003a, 2003b; Liszt 2014a; Lee et al. 2015); though, the fraction missed in some localized regions may be much higher (Bihr et al. 2015).

Since dust and gas are generally well mixed, absorption due to dust grains has been widely used as a proxy for total gas column density. Early work (e.g., Savage \& Jenkins 1972, Bohlin et al. 1978) observed Ly $\alpha$ and $\mathrm{H}_{2}$ absorption in stellar spectra to calibrate the relationship between total hydrogen column density $N_{\mathrm{H}}$, and the color excess $E(B-V)$. Similar work was carried out by comparing X-ray absorption with optical extinction, $A_{V}$ (Reina \& Tarenghi 1973, Gorenstein 1975). Bohlin et al's value of $N_{\mathrm{H}} / E(B-V)=$ $5.8 \times 10^{21} \mathrm{~cm}^{-2} \mathrm{mag}^{-1}$ has become a widely accepted standard.

Dust emission is also a powerful tool and requires no background source population. The dust emission spectrum in the bulk of the ISM peaks in the FIR-to-millimeter range, and arises mostly from large grains in thermal equilibrium with the ambient local radiation field (Draine 2003, Draine \& Li 2007). It has long been recognized that FIR dust emission could potentially be a better tracer of $N_{\mathrm{H}}$ than $\mathrm{HI}$ and CO (de Vries et al. 1987, Heiles et al. 1988, Blitz et al. 1990; Reach et al. 1994). An excess of dust intensity and/or optical depth above a linear correlation with $N_{\mathrm{H}}$ (as measured by $\mathrm{H}$ I and $\mathrm{CO}$ ) is typically found in the range $A_{V}=0.3-2.7 \mathrm{mag}$ (Planck Collaboration et al. 2011, 2014a, 2014b; Martin et al. 2012), consistent with the range where CO-dark $\mathrm{H}_{2}$ can exist. Alternative explanations cannot be definitively ruled out, however. These include (1) the evolution of dust grains across the gas phases, (2) underestimation of the total gas column due to significant cold H I opacity, and (3) insufficient sensitivity for $\mathrm{CO}$ detection. It has also been impossible to rule out remaining systematic effects in the Planck data or bias in the estimate of $\tau_{353}$ introduced by the choice of the modified blackbody model.

In this study, we examine the correlations between accurately derived $\mathrm{H}$ I column densities and dust-based proxies for $N_{\mathrm{H}}$. We make use of opacity-corrected H I column densities derived from two surveys: the Arecibo Millennium Survey (MS, Heiles \& Troland 2003b, hereafter HT03), and 21SPONGE (Murray et al. 2015), both of which used on-/offsource measurements toward extragalactic radio continuum sources to derive accurate physical properties for the atomic ISM. We also make use of archival $\mathrm{OH}$ data from the Millennium Survey, recently published for the first time in a companion paper, $\mathrm{Li}$ et al. (2018). $\mathrm{OH}$ is an effective tracer of diffuse molecular regions (Wannier et al. 1993; Liszt \& Lucas 1996; Barriault et al. 2010; Allen et al. 2012, 2015; Xu et al. 2016; Li et al. 2018), and has recently been surveyed at high sensitivity in parts of the Galactic plane (Dawson et al. 2014; Bihr et al. 2015). There exists both theoretical and observational evidence for the close coexistence of interstellar $\mathrm{OH}$ and $\mathrm{H}_{2}$. Observationally, they appear to reside in the same environments, as evidenced by tight relations between their column densities (Weselak \& Krełowski 2014). Theoretically, the synthesis of $\mathrm{OH}$ is driven by the ions $\mathrm{O}^{+}$and $\mathrm{H}_{3}^{+}$but requires $\mathrm{H}_{2}$ as the precursor; once $\mathrm{H}_{2}$ becomes available, $\mathrm{OH}$ can be formed efficiently through the chargeexchange chemical reactions initiated by cosmic-ray ionization (van Dishoeck \& Black 1986). Here we combine H I, OH, and dust data sets to obtain new measurements of the abundance ratio, $X_{\mathrm{OH}}=N_{\mathrm{OH}} / N_{\mathrm{H}_{2}}$ - a key quantity for the interpretation of $\mathrm{OH}$ data sets.

The structure of this article is as follows. In Section 2, the observations, the data processing techniques, and corrections on $\mathrm{H}$ I are briefly summarized. In Section 3, the results from $\mathrm{OH}$ observations are discussed. Section 4 discusses the relationship between $\tau_{353}, E(B-V)$ and $N_{\mathrm{H}}$ in the atomic ISM. We finally estimate the $\mathrm{OH} / \mathrm{H}_{2}$ abundance ratio in Section 5 before concluding in Section 6.

\section{Data Sets}

In this study, we use the all-sky optical depth $\left(\tau_{353}\right)$ map of the dust model data measured by Planck/IRAS (Planck Collaboration et al. 2014a-hereafter PLC2014a), the reddening $E(B-V)$ all-sky map from Green et al. (2018), H I data from both the 21-SPONGE Survey (Murray et al. 2015) and the Millennium Survey (Heiles \& Troland 2003a, HT03), $\mathrm{OH}$ data from the Millennium Survey (Li et al. 2018), and CO data from the Delingha $14 \mathrm{~m}$ Telescope, the Caltech Submillimeter Observatory (CSO), and the IRAM $30 \mathrm{~m}$ telescope (Li et al. 2018).

\section{1. $\mathrm{HI}$ and $\mathrm{OH}$}

$\mathrm{H}$ I data from the Millennium Arecibo $21 \mathrm{~cm}$ AbsorptionLine Survey (hereafter MS) was taken toward 79 strong radio sources (typically $S \gtrsim 2$ Jy) using the Arecibo L-wide receiver. The two main lines of ground state $\mathrm{OH}$ at 1665.402 and $1667.359 \mathrm{MHz}$ were observed simultaneously toward 72 positions, and $\mathrm{OH}$ absorption was detected along 19 of these sightlines (see also Li et al. 2018). The observations are described in detail by HT03. Briefly, their so-called Z16 observation pattern consists of one on-source absorption spectrum toward the background radio source and 16 offsource emission spectra with the innermost positions at 1.0 HPBW and the outermost positions at $\sqrt{2}$ HPBW from the central source. The off-source "expected" emission spectrum, the emission profile we would observe in the absence of the continuum source, is then estimated by modeling the 17-point measurements. In this work, we use the published values of HT03 for the total $\mathrm{H}$ I column density, $N_{\mathrm{H} \text { I }}$ (scaled as described below), and use the off-source (expected) MS emission profiles to compute the H I column density under the optically thin assumption, $N_{\mathrm{H}}^{*}$, where required. We compute $\mathrm{OH}$ column densities ourselves, as described in Section 3. All OH emission and absorption spectra are scaled to a main-beam temperature scale using a beam efficiency of $\eta_{b}=0.5$ (Heiles et al. 2001), 


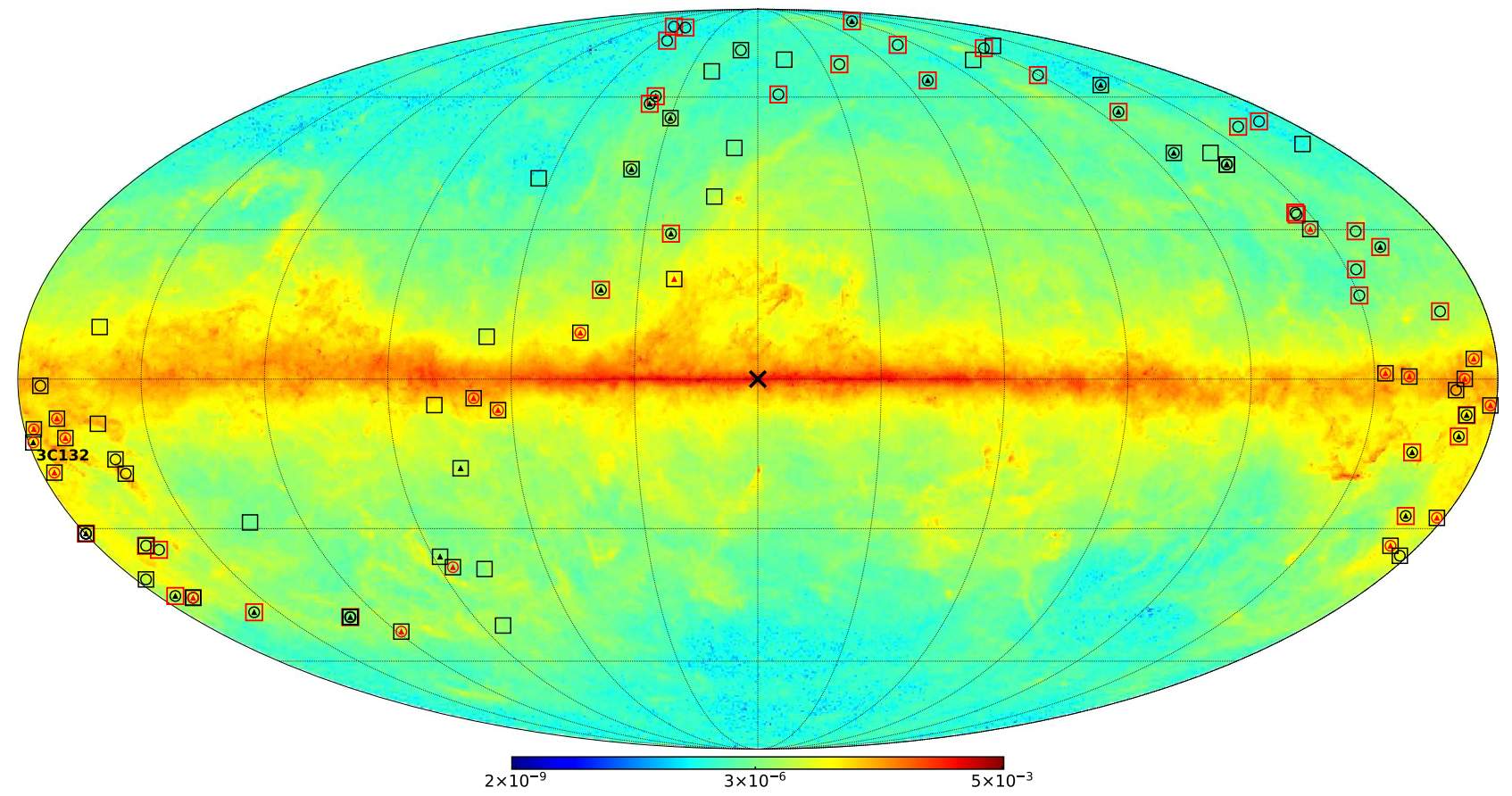

Figure 1. Locations of all 93 sightlines considered in this study, overlaid on the map of dust optical depth $\tau_{353}$. Squares show H I absorption detections (93/93); red circles show OH absorption detections (19/72); black circles show nondetections (51/72); red triangles show CO detections (19/44); and black triangles show nondetections (25/44). For purely atomic sightlines (those with no molecular detection at the threshold discussed in Section 4), the squares are colored red. Note that the absence of a symbol indicates that the sightline was not observed in that particular tracer. The labeled sightline toward 3C132 (far left) shows the single position detected in $\mathrm{H} \mathrm{I}$ and $\mathrm{OH}$ but not detected in $\mathrm{CO}$ emissions. The "X" marker labels the center of the Milky Way. Note that the symbols for a small number of sightlines entirely overlap due to their proximity on the sky.

appropriate if the $\mathrm{OH}$ is not extended compared to the Arecibo beam size of $3^{\prime}$.

In order to increase the source sample, we also use H I data from the Very Large Array (VLA) 21-SPONGE Survey, which observed 30 continuum sources, including 16 in common with the Millennium Survey sample (Murray et al. 2015). 21SPONGE used on-source absorption data from the VLA, combining them with off-source emission profiles observed with Arecibo. Murray et al. (2015) report an excellent agreement between the optical depths measured by the two surveys, demonstrating that the single dish Arecibo absorption profiles are not significantly contaminated with resolved $21 \mathrm{~cm}$ emission. Note that in this work we have used an updated scaling of the 21-SPONGE emission profiles, which applies a beam efficiency factor of 0.94 to the Arecibo spectra. The total number of unique sightlines presented in this work is therefore 93. The locations of all observed sources in Galactic coordinates are presented in Figure 1. Where sources were observed in both the MS and 21-SPONGE, we use the MS data.

\subsubsection{H I Intensity Scale Corrections}

We check our $N_{\mathrm{H}}^{*}$ against the Leiden-Argentine-Bonn survey (LAB, Hartmann \& Burton 1997; Kalberla et al. 2005) and the HI4PI survey (HI4PI Collaboration et al. 2016). Both are widely regarded as a gold standard in the absolute calibration of Galactic HI. We find that the optically thin column densities derived from 21-SPONGE are consistent with LAB and HI4PI. However, the MS values are systematically lower than both LAB and HI4PI by a factor of $\sim 1.14$. A possible explanation for this difference lies in the fact that (in contrast to 21-SPONGE) the MS did not apply a main-beam efficiency.

To bring the MS data set in-line with LAB, HI4PI, and 21SPONGE, one might assume that both the on-source and offsource spectra must be rescaled, and the opacity-corrected column densities recomputed according to the method of HT03 (or equivalent). However, $N_{\mathrm{H}}$ may in fact be obtained from the tabulated values of HT03, with no need to perform a full reanalysis of the data. For warm components, the tabulated values of $N_{\mathrm{H}}$ are simply scaled by 1.14 -appropriate since these were originally computed directly from the the integrated off-source (expected) profiles under the optically thin assumption. For cold components, we recall that the radiative transfer equations for the on-source and off-source (expected) spectra in the MS data set are given by:

$$
\begin{gathered}
T_{\mathrm{B}}^{\mathrm{ON}}(v)=\left(T_{\mathrm{bg}}+T_{\mathrm{c}}\right) e^{-\tau_{v}}+T_{\mathrm{s}}\left(1-e^{-\tau_{v}}\right)+T_{\mathrm{rx}} \\
T_{\mathrm{B}}^{\mathrm{OFF}}(v)=T_{\mathrm{bg}} e^{-\tau_{v}}+T_{\mathrm{s}}\left(1-e^{-\tau_{v}}\right)+T_{\mathrm{rx}},
\end{gathered}
$$

where $T_{\mathrm{B}}^{\mathrm{OFF}}(v)$ and $T_{\mathrm{B}}^{\mathrm{ON}}(v)$ are the main-beam temperatures of the off-source spectrum and on-source spectrum, respectively. $T_{\mathrm{S}}$ is the spin temperature, $\tau_{v}$ is the optical depth, $T_{\mathrm{rx}}$ is the receiver temperature $(\sim 25 \mathrm{~K})$, and $T_{\mathrm{c}}$ is the main-beam temperature of the continuum source, obtained from the linefree portions of the on-source spectrum. $T_{\mathrm{bg}}$ is the continuum background brightness temperature including the $2.7 \mathrm{~K}$ isotropic radiation from $\mathrm{CMB}$ and the Galactic synchrotron background at the source position. Equations (1) and (2) may be solved for $\tau_{v}$ and $T_{\mathrm{s}}$ :

$$
e^{-\tau_{v}}=\frac{T_{\mathrm{B}}^{\mathrm{ON}}(v)-T_{\mathrm{B}}^{\mathrm{OFF}}(v)}{T_{\mathrm{c}}},
$$




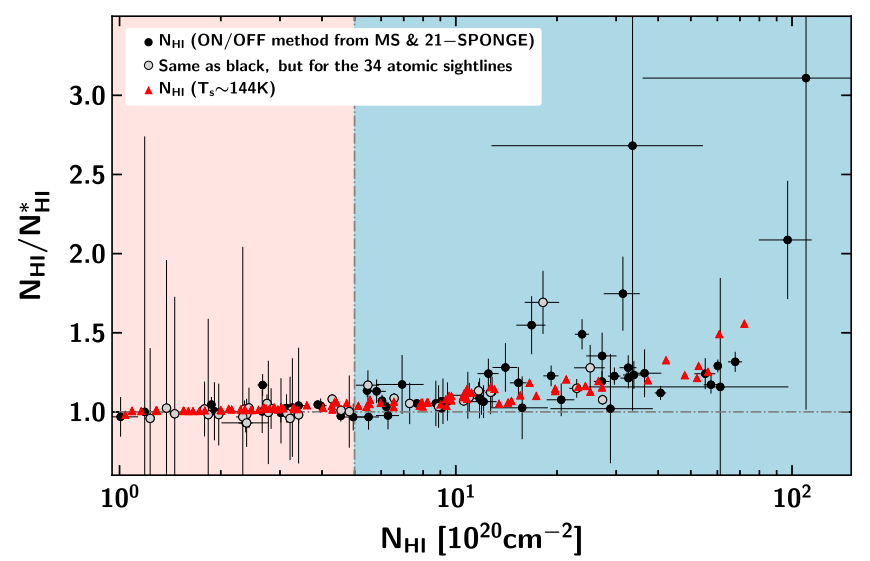

Figure 2. Ratio $f=N_{\mathrm{H} \mathrm{I}} / N_{\mathrm{H} \text { I }}^{*}$ as a function of opacity-corrected $N_{\mathrm{H} \text { I }}$ along 93 sightlines from the MS and 21-SPONGE surveys. Circles show accurate $N_{\mathrm{H}}$ obtained via on- and off-source observations (HT03; scaled as described in the text), with the 34 atomic sightlines (selection criteria described in Section 4) filled gray and all other points filled black. Red triangles show $N_{\mathrm{H} \text { I }}$ obtained from $N_{\mathrm{H}}^{*}$ assuming a single isothermal component of $T_{\mathrm{s}} \sim 144 \mathrm{~K}$. The vertical dashed line is plotted at $N_{\mathrm{H} \mathrm{I}}=5 \times 10^{20} \mathrm{~cm}^{-2}$; the horizontal dashed line marks where $N_{\mathrm{H} \mathrm{I}}=N_{\mathrm{H} \text { I }}^{*}$

$$
T_{\mathrm{s}}=\frac{T_{\mathrm{B}}^{\mathrm{OFF}}(v)-T_{\mathrm{bg}} e^{-\tau_{v}}-T_{\mathrm{rx}}}{1-e^{-\tau_{v}}} .
$$

From Equation (3), it is clear that optical depth is unchanged by any rescaling, which will affect both the numerator and denominator of the expression identically. Only $T_{s}$ must be recomputed. This is done on a component-by-component basis from the tabulated Gaussian fit parameters for peak optical depth, $\tau_{0}$, peak brightness temperature (scaled by 1.14), and the linewidth $\Delta v$. The corrected $N_{\mathrm{H}}$ is obtained from

$$
\frac{N_{\mathrm{H} \mathrm{I}}}{\left[10^{18} \mathrm{~cm}^{-2}\right]}=1.94 \cdot \tau_{0} \cdot \frac{T_{\mathrm{S}}}{[\mathrm{K}]} \cdot \frac{\Delta v}{\left[\mathrm{~km} \mathrm{~s}^{-1}\right]},
$$

where the factor 1.94 includes the usual constant 1.8224 and the 1.065 arising from the integration over the Gaussian line profile.

\subsection{2. $\mathrm{N}_{H_{I}}$ versus $N_{\mathrm{H} I}^{*}$}

We show in Figure 2 the correlation between $N_{\mathrm{H} \text { I }}$ and $N_{\mathrm{H} \text { I }}^{*}$ toward all 93 positions. While optically thin H I column density is comparable with the true column density in diffuse/lowdensity regions with $N_{\mathrm{H}} \lesssim 5 \times 10^{20} \mathrm{~cm}^{-2}$, opacity effects start to become apparent above $\sim 5 \times 10^{20} \mathrm{~cm}^{-2}$.

If a linear fit is performed to the data, the ratio $f=N_{\mathrm{H}_{\mathrm{I}}} / N_{\mathrm{H} \text { I }}^{*}$ may be described as a function of $\log \left(N_{\mathrm{H}_{\mathrm{I}}}^{*} / 10^{20}\right)$ with a slope of $(0.19 \pm 0.02)$ and an intercept of $(0.89 \pm 0.02)$ (see also Lee et al. 2015). Alternatively, a simple isothermal correction to the optically thin $N_{\mathrm{H} \text { I }}^{*}$ data with $T_{\mathrm{s}} \sim 144 \mathrm{~K}$ also yields a good agreement with our data points, as illustrated in Figure 2 (see also Liszt 2014b). This approach also better fits the low $N_{\mathrm{H}}$ plateau, $\quad N_{\mathrm{H} \mathrm{I}}<5 \times 10^{20} \mathrm{~cm}^{-2}$, below which $N_{\mathrm{H} \mathrm{I}}^{*} \approx N_{\mathrm{H} \mathrm{I}}$. While a single component with a constant spin temperature is a poor physical description of interstellar H I, it can provide a reasonable (if crude) correction for opacity.

\section{2. $\mathrm{CO}$}

As described in $\mathrm{Li}$ et al. (2018), a CO follow-up survey was conducted toward 44 of the sightlines considered in this work.
The $J=1-0$ transitions of ${ }^{12} \mathrm{CO},{ }^{13} \mathrm{CO}$, and $\mathrm{C}^{18} \mathrm{O}$ were observed with the Delingha $13.7 \mathrm{~m}$ telescope in China. ${ }^{12} \mathrm{CO}(J=2-1)$ data for 45 sources and $J=3-2$ data for 8 sources with strong ${ }^{12} \mathrm{CO}$ emission were taken with the $10.4 \mathrm{~m}$ CSO on Maunakea, with further supplementary data obtained by the IRAM $30 \mathrm{~m}$ telescope. In this work, we use $\mathrm{CO}$ data solely to identify and exclude from some parts of the analysis positions with detected CO-bright molecular gas-comprising 19 of the 44 observed positions. These positions are identified in Figure 1.

\subsection{Dust}

To trace the total gas column density $N_{\mathrm{H}}$, we use publicly available all-sky maps of the $353 \mathrm{GHz}$ dust optical depth $\left(\tau_{353}\right)$ from the Planck satellite. The $\tau_{353}$ map was obtained by a modified blackbody (MBB) fit to the first 15 months of 353, 545 , and $857 \mathrm{GHz}$ data, together with IRAS 100 micron data (for details, see PLC2014a). The angular resolution of this data set is 5 arcmin. In this work, we use the R1.20 data release in Healpix ${ }^{15}$ format (Górski et al. 2005). For dust reddening, we employ the newly released all-sky 3D dust map of Green et al. (2018) at an angular resolution of 3!4-13!7, which was derived from 2MASS and the latest Pan-STARRS 1 data photometry. In contrast to emission-based dust maps that depend on the modeling of the temperature, optical depth, and the shape of the emission spectrum, in maps based on stellar photometry reddening values are more directly measured and not contaminated from zodiacal light or large-scale structure. Here we convert the Green et al. (2018) Bayestar17 dust map to $E(B-V)$ by applying a scaling factor of 0.884 , as described in the documentation accompanying the data release. ${ }^{16}$

\section{OH Data Analysis}

The Millennium Survey $\mathrm{OH}$ data consists of on-source and off-source "expected" spectra for each of the $\mathrm{OH}$ lines. In our companion paper ( $\mathrm{Li}$ et al. 2018), we use the method of HT03 to derive $\mathrm{OH}$ optical depths, excitation temperatures and column densities. Namely, we obtain solutions for the excitation temperature, $T_{\mathrm{ex}}$, and $\tau$ via Gaussian fitting (to both the on-source and off-source spectra) that explicitly includes the appropriate treatment of the radiative transfer. In the present work, we use a simpler channel-by-channel method for the derivation of $T_{\mathrm{ex}}$.

The radiative transfer equations for the on-source and offsource (expected) spectra are identical to those for H I, given above in Equations (1) and (2). All terms and their meanings are identical, with the exception that the spin temperature, $T_{\mathrm{s}}$ is replaced by $T_{\mathrm{ex}} . T_{\mathrm{bg}}$ is the continuum background brightness temperature including the $2.7 \mathrm{~K}$ isotropic radiation from $\mathrm{CMB}$ and the Galactic synchrotron background at the source position. For consistency with HT03 and Li et al. (2018), we estimate the synchrotron contribution at 1665.402 and $1667.359 \mathrm{MHz}$ from the $408 \mathrm{MHz}$ continuum map of Haslam et al. (1982), by adopting a temperature spectral index of 2.8 , such that

$$
T_{\mathrm{bg}}=2.7+T_{\mathrm{bg}, 408}\left(\nu_{\mathrm{OH}} / 408\right)^{-2.8},
$$

resulting in typical values of around $3.5 \mathrm{~K}$. The background continuum contribution from Galactic H II regions may be

\footnotetext{
15 http://healpix.sourceforge.net

16 http://argonaut.skymaps.info/usage
} 

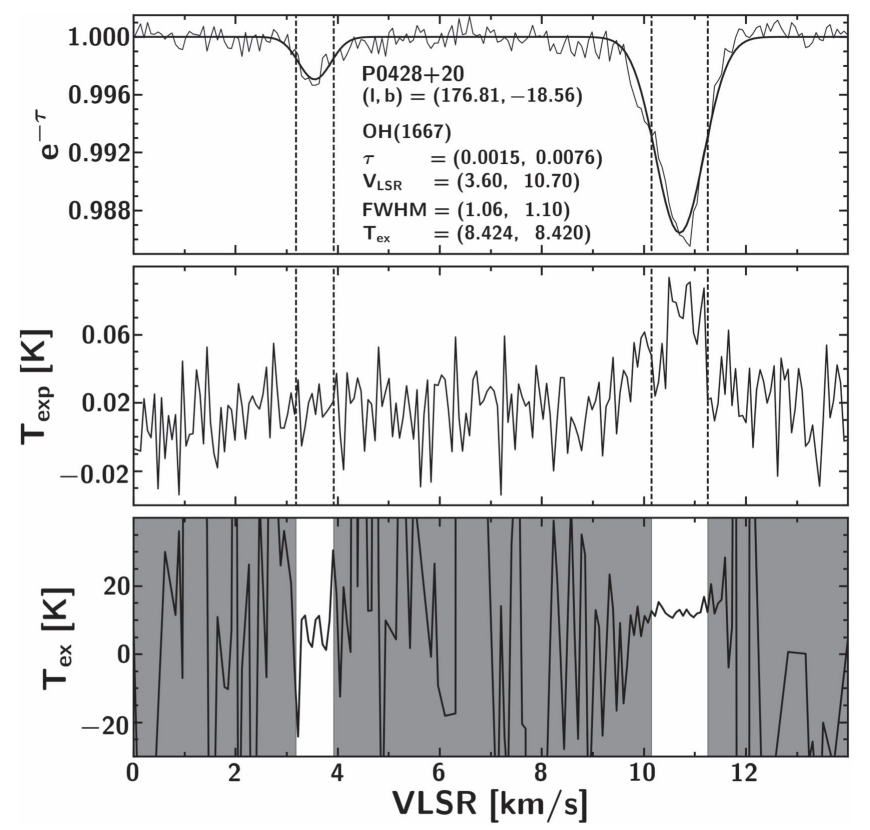

Figure 3. Example of $\mathrm{OH} 1667 \mathrm{MHz} e^{-\tau_{v}}$ (top), expected $T_{\mathrm{B}}^{\mathrm{OFF}}$ (middle), and $T_{\text {ex }}$ (bottom) spectra for the source P0428+20. The FWHM of the Gaussian fits to the absorption profile are used to define the range over which $T_{\mathrm{ex}}$ is computed for each component, shown as white regions in the bottom panel.

safely ignored, since the continuum sources we observed are either at high Galactic latitudes or Galactic anti-center longitudes. Thus, in line-free portions of the off-source spectra:

$$
T_{\mathrm{B}}^{\mathrm{OFF}}(v)=T_{\mathrm{bg}}+T_{\mathrm{rx}} \text {. }
$$

In the absence of information about the true gas distribution, we assume that $\mathrm{OH}$ clouds cover fully both the continuum source and the main beam of the telescope. We may therefore solve Equations (1) and (2) to derive $T_{\mathrm{ex}}$ and $\tau_{v}$ for each of the $\mathrm{OH}$ lines, as shown in Equations (3) and (4) for the case of $\mathrm{H} \mathrm{I}$.

We fit each $\mathrm{OH}$ opacity spectrum (cf. Equation (3)) with a set of Gaussian profiles to obtain the peak optical depth $\left(\tau_{0, n}\right)$, central velocity $\left(v_{0, n}\right)$, and FWHM $\left(\Delta v_{n}\right)$ of each component, $n$. Equation (4) is then used to calculate excitation temperature spectra. Examples of $e^{-\tau_{v}}, T_{\mathrm{B}}^{\mathrm{OFF}}$, and $T_{\mathrm{ex}}$ spectra are shown in Figure 3, together with their associated Gaussian fits. It can be seen that the $T_{\text {ex }}$ spectra are approximately flat within the FWHM of each Gaussian component. We therefore compute an excitation temperature for each component from the mean $T_{\mathrm{ex}}$ in the range $v_{0, n} \pm \Delta v / 2$.

Figure 4 compares the $\tau_{0}$ and $T_{\text {ex }}$ values obtained from our method with those of Li et al. (2018), demonstrating that the two methods generally return consistent results. Minor differences arise only for the most complex sightlines through the Galactic plane $(\mathrm{G} 197.0+1.1$, T0629+10), where the spectra are not simple to analyze; however, even these points are mostly consistent to within the errors.

We compute total $\mathrm{OH}$ column densities, $N_{\mathrm{OH}}$, independently from both the 1667 and $1665 \mathrm{MHz}$ lines via:

$$
\begin{aligned}
& \frac{N_{\mathrm{OH}, 1667}}{\left[10^{14} \mathrm{~cm}^{-2}\right]}=2.39 \cdot \tau_{1667} \cdot \frac{T_{\mathrm{ex}, 1667}}{[\mathrm{~K}]} \cdot \frac{\Delta v_{1667}}{\left[\mathrm{~km} \mathrm{~s}^{-2}\right]}, \\
& \frac{N_{\mathrm{OH}, 1665}}{\left[10^{14} \mathrm{~cm}^{-2}\right]}=4.30 \cdot \tau_{1665} \cdot \frac{T_{\mathrm{ex}, 1665}}{[\mathrm{~K}]} \cdot \frac{\Delta v_{1665}}{\left[\mathrm{~km} \mathrm{~s}^{-2}\right]},
\end{aligned}
$$
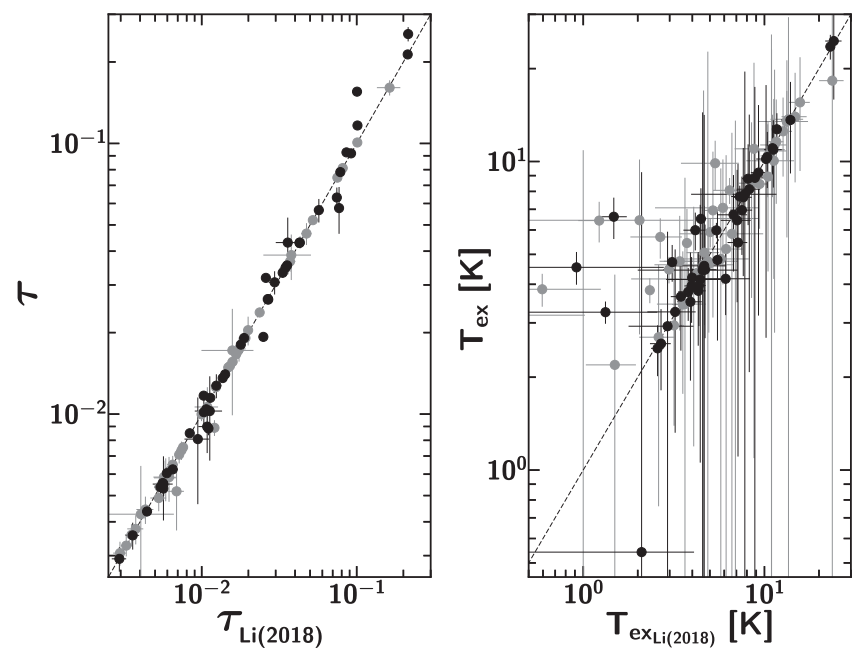

Figure 4. Comparison between derived values of the peak optical depth $\tau$ (left panel), and $T_{\text {ex }}$ (right panel) for both $\mathrm{OH}$ main lines, $1667 \mathrm{MHz}$ (black) and $1665 \mathrm{MHz}$ (gray), as obtained from our companion paper by Li et al. (2018) and the present work. The dashed lines mark where the two values are equal.

where the constants include Einstein $A$-coefficients of $A_{1667}=7.778 \times 10^{-11} \mathrm{~s}^{-1}$ and $A_{1665}=7.177 \times 10^{-11} \mathrm{~s}^{-1}$ for the $\mathrm{OH}$ main lines (Destombes et al. 1977). All values of $\tau_{0}, T_{\mathrm{ex}}$, and $N_{\mathrm{OH}}$ are tabulated in Table 1.

\section{Dust-based Proxies for Total Neutral Gas Column Density}

In this section, we will investigate the correlations between dust properties and the total gas column density $N_{\mathrm{H}}$. Specifically, we consider dust optical depth at $353 \mathrm{GHz}, \tau_{353}$, and reddening, $E(B-V)$, with data sets sourced as described in Section 2.3. When these quantities are used as proxies for $N_{\mathrm{H}}$, a single linear relationship between the measured quantity and $N_{\mathrm{H}}$ is typically assumed. In this work, our $\mathrm{HI}$ data set provides accurate (opacity-corrected) atomic column densities, while complementary $\mathrm{OH}$ and $\mathrm{CO}$ data allow us to identify and exclude sightlines with molecular gas (dark or not). We are therefore able to measure $\tau_{353} / N_{\mathrm{H}}$ and $E(B-V) / N_{\mathrm{H}}$ along a sample of purely atomic sightlines for which $N_{\mathrm{H}}$ is very well constrained.

In the following, we consider 34/93 sightlines to be "purely atomic." These are defined as either (a) sightlines where CO and $\mathrm{OH}$ were observed and not detected in emission (16/93), or (b) sightlines where $\mathrm{CO}$ was not observed but $\mathrm{OH}$ was observed but not detected (18/93 sightlines). In both cases, we require that $\mathrm{OH}$ be undetected in the $1667 \mathrm{MHz}$ line to a detection limit of $N_{\mathrm{OH}}<1 \times 10^{13} \mathrm{~cm}^{-2}$ (see Li et al. 2018), which excludes some positions with weaker continuum background sources. We may confidently assume that these sightlines contain very little or no $\mathrm{H}_{2}$ and note that all but one of them lie outside the Galactic plane $\left(|b|>10^{\circ}\right)$. Figure 5 shows maps of the immediate vicinity of these sightlines in $\tau_{353}$ and $E(B-V)$. Identical maps for the 19 sightlines with $\mathrm{OH}$ detections (see also Section 5), are shown in Figure 6.

In all of the following subsections, $N_{\mathrm{H}}$ is taken to be equal to $N_{\mathrm{H} \text { I }}$, the opacity-corrected $\mathrm{H}$ I column density, as derived along sightlines with no molecular gas detected in emission. 
Table 1

Parameters for $\mathrm{OH}$ Main Lines

\begin{tabular}{|c|c|c|c|c|c|c|c|c|c|c|c|}
\hline \multirow{2}{*}{$\begin{array}{l}\text { Source } \\
\text { (Name) }\end{array}$} & \multirow{2}{*}{$\begin{array}{l}l / b \\
\left({ }^{\circ}\right)\end{array}$} & \multicolumn{5}{|c|}{ OH(1665) } & \multicolumn{5}{|c|}{ OH(1667) } \\
\hline & & $\tau$ & $\begin{array}{c}V_{\mathrm{lsr}} \\
\left(\mathrm{km} \mathrm{s}^{-1}\right)\end{array}$ & $\begin{array}{c}\Delta V \\
\left(\mathrm{~km} \mathrm{~s}^{-1}\right)\end{array}$ & $\begin{array}{l}T_{\mathrm{ex}} \\
(\mathrm{K})\end{array}$ & $\begin{array}{c}N(\mathrm{OH}) \\
\left(10^{14} \mathrm{~cm}^{-2}\right)\end{array}$ & $\tau$ & $\begin{array}{c}V_{\mathrm{lsr}} \\
\left(\mathrm{km} \mathrm{s}^{-1}\right)\end{array}$ & $\begin{array}{c}\Delta V \\
\left(\mathrm{~km} \mathrm{~s}^{-1}\right)\end{array}$ & $\begin{array}{l}T_{\mathrm{ex}} \\
(\mathrm{K})\end{array}$ & $\begin{array}{c}N(\mathrm{OH}) \\
\left(10^{14} \mathrm{~cm}^{-2}\right)\end{array}$ \\
\hline $3 \mathrm{C} 105$ & $187.6 /-33.6$ & $0.0156 \pm 0.0003$ & $8.14 \pm 0.01$ & $0.95 \pm 0.03$ & $4.65 \pm 1.86$ & $0.29 \pm 0.12$ & $0.0265 \pm 0.0004$ & $8.17 \pm 0.01$ & $0.94 \pm 0.02$ & $3.95 \pm 0.95$ & $0.23 \pm 0.06$ \\
\hline $3 \mathrm{C} 105$ & $7.6 /-33.6$ & $0.0062 \pm 0.0003$ & $10.22 \pm 0.02$ & $0.93 \pm 0.06$ & $8.5 \pm 4.89$ & $21 \pm 0.12$ & $0.0104 \pm 0.0004$ & $10.25 \pm 0.02$ & $0.96 \pm 0.04$ & $7.66 \pm 3.41$ & $18 \pm 0.08$ \\
\hline $3 \mathrm{C} 109$ & $181.8 /-27.8$ & $0.0023 \pm 0.0003$ & $9.15 \pm 0.11$ & $1.03 \pm 0.26$ & $18.28 \pm 27.06$ & $0.18 \pm 0.27$ & $0.0036 \pm 0.0004$ & $9.24 \pm 0.05$ & $0.75 \pm 0.12$ & $24.58 \pm 8.7$ & $0.16 \pm 0.06$ \\
\hline $3 \mathrm{C} 109$ & $181.8 /-27.8$ & $0.0036 \pm 0.0003$ & $10.45 \pm 0.07$ & $0.98 \pm 0.15$ & $13.97 \pm 5.41$ & $0.21 \pm 0.09$ & $0.0053 \pm 0.0004$ & $10.55 \pm 0.04$ & $1.02 \pm 0.1$ & $13.63 \pm 4.48$ & $0.18 \pm 0.06$ \\
\hline $3 \mathrm{C} 123$ & $170.6 /-11.7$ & $0.0191 \pm 0.0007$ & $3.65 \pm 0.06$ & $1.19 \pm 0.11$ & $10.92 \pm 3.26$ & $1.05 \pm 0.33$ & $0.0348 \pm 0.0009$ & $3.71 \pm 0.04$ & $1.22 \pm 0.07$ & $10.92 \pm 2.69$ & $1.1 \pm 0.28$ \\
\hline $3 \mathrm{C} 123$ & $170.6 /-11.7$ & $0.0431 \pm 0.0023$ & $4.43 \pm 0.01$ & $0.53 \pm 0.03$ & $8.06 \pm 0.78$ & $0.78 \pm 0.09$ & $0.0919 \pm 0.0029$ & $4.46 \pm 0.0$ & $0.53 \pm 0.01$ & $7.7 \pm 0.65$ & $0.89 \pm 0.08$ \\
\hline $3 \mathrm{C} 123$ & $170.6 /-11.7$ & $0.0337 \pm 0.0008$ & $5.37 \pm 0.01$ & $0.91 \pm 0.03$ & $11.59 \pm 4.3$ & $1.53 \pm 0.57$ & $0.0784 \pm 0.0009$ & $5.47 \pm 0.01$ & $0.92 \pm 0.01$ & $8.79 \pm 2.57$ & $1.5 \pm 0.44$ \\
\hline $3 \mathrm{C} 131$ & $171.4 /-7.8$ & $0.0065 \pm 0.0005$ & $4.55 \pm 0.02$ & $0.56 \pm 0.05$ & $12.52 \pm 3.59$ & $0.19 \pm 0.06$ & $0.0117 \pm 0.0004$ & $4.64 \pm 0.01$ & $0.78 \pm 0.04$ & $6.96 \pm 1.98$ & $0.15 \pm 0.04$ \\
\hline $3 \mathrm{C} 131$ & $171.4 /-7.8$ & $0.0073 \pm 0.0006$ & $6.81 \pm 0.06$ & $2.91 \pm 0.23$ & $8.94 \pm 4.54$ & $0.82 \pm 0.42$ & $0.0089 \pm 0.0005$ & $5.84 \pm 0.03$ & $0.67 \pm 0.08$ & $11.04 \pm 2.07$ & $0.16 \pm 0.04$ \\
\hline $3 \mathrm{C} 131$ & $171.4 /-7.8$ & $0.0166 \pm 0.0007$ & $6.59 \pm 0.01$ & $0.42 \pm 0.02$ & $5.69 \pm 0.85$ & $0.17 \pm 0.03$ & $0.0319 \pm 0.0007$ & $6.55 \pm 0.01$ & $0.45 \pm 0.02$ & $5.99 \pm 0.84$ & $0.2 \pm 0.03$ \\
\hline $3 \mathrm{C} 131$ & $171.4 /-7.8$ & $0.0521 \pm 0.0007$ & $7.23 \pm 0.0$ & $0.55 \pm 0.01$ & $5.91 \pm 0.64$ & $0.72 \pm 0.08$ & $0.0927 \pm 0.0$ & $7.22 \pm 0.0$ & $0.65 \pm 0.01$ & $5.98 \pm 0.34$ & $.85 \pm 0.05$ \\
\hline $3 \mathrm{C} 132$ & $178.9 /-12.5$ & $0.0033 \pm 0.0003$ & $7.82 \pm 0.04$ & $0.9 \pm 0.1$ & $15.55 \pm 6.23$ & $0.19 \pm 0.08$ & $0.0056 \pm 0.0003$ & $7.79 \pm 0.02$ & $0.79 \pm 0.06$ & $23.56 \pm 2.17$ & $0.25 \pm 0.03$ \\
\hline $3 \mathrm{C} 133$ & $177.7 /-9.9$ & $0.1008 \pm 0.001$ & $7.66 \pm 0.0$ & $0.53 \pm 0.0$ & $4.47 \pm 0.44$ & $1.01 \pm 0.1$ & $0.2132 \pm 0.0014$ & $7.68 \pm 0.0$ & $0.52 \pm 0.0$ & $3.25 \pm 0.27$ & $0.85 \pm 0.07$ \\
\hline $3 \mathrm{C} 133$ & $177.7 /-9.9$ & $0.0149 \pm 0.001$ & $7.94 \pm 0.02$ & $1.22 \pm 0.04$ & $7.08 \pm 3.08$ & $0.55 \pm 0.24$ & $0.0333 \pm 0.0013$ & $7.96 \pm 0.01$ & $1.23 \pm 0.02$ & $4.17 \pm 0.99$ & $0.4 \pm 0.1$ \\
\hline $3 \mathrm{C} 154$ & $185.6 / 4.0$ & $0.0266 \pm 0.0006$ & $-2.32 \pm 0.02$ & $0.74 \pm 0.03$ & $2.69 \pm 1.93$ & $0.23 \pm 0.16$ & $0.0429 \pm 0.0006$ & $-2.34 \pm 0.01$ & $0.71 \pm 0.02$ & $2.57 \pm 0.75$ & $0.19 \pm 0.05$ \\
\hline $3 \mathrm{C} 154$ & $185.6 / 4.0$ & $0.01 \pm 0.0006$ & $-1.39 \pm 0.04$ & $0.83 \pm 0.09$ & $5.2 \pm 5.28$ & $0.18 \pm 0.19$ & $0.0181 \pm 0.0005$ & $-1.34 \pm 0.02$ & $0.94 \pm 0.05$ & $4.46 \pm 1.79$ & $0.18 \pm 0.07$ \\
\hline $3 \mathrm{C} 154$ & $5.6 / 4.0$ & $.0038 \pm 0.0005$ & $2.23 \pm 0.07$ & $1.14 \pm 0.17$ & $5.83 \pm 6.56$ & $011+012$ & $0.0054 \pm 0.0004$ & $2.19 \pm 0.05$ & $1.57 \pm 0.13$ & $0.54 \pm 8.69$ & $0.01 \pm 0.17$ \\
\hline $3 \mathrm{C} 167$ & $207.3 / 1.2$ & $0.0106 \pm 0.0019$ & $18.46 \pm 0.12$ & $1.49 \pm 0.35$ & $4.75 \pm 17.95$ & $0.32 \pm 1.22$ & $0.009 \pm 0.0018$ & $17.77 \pm 0.15$ & $1.76 \pm 0.49$ & $4.59 \pm 9.57$ & $0.17 \pm 0.36$ \\
\hline $3 \mathrm{C} 18$ & $118.6 /-52.7$ & $0.0031 \pm 0.0003$ & $-8.52 \pm 0.11$ & $2.64 \pm 0.27$ & $10.92 \pm 14.88$ & $0.38 \pm 0.52$ & $0.006 \pm 0.0003$ & $-8.34 \pm 0.05$ & $2.61 \pm 0.14$ & $9.2 \pm 6.04$ & $0.34 \pm 0.23$ \\
\hline $3 \mathrm{C} 18$ & $3.6 /-52.7$ & $0.0056 \pm 0.0004$ & $-7.82 \pm 0.02$ & $0.67 \pm 0.07$ & $6.45 \pm 3.7$ & & $0.0079 \pm 0$. & $-7.85 \pm 0.01$ & $0.6 \pm 0.04$ & $4.83 \pm 1.6$ & $0.05 \pm 0.02$ \\
\hline $3 \mathrm{C} 207$ & $213.0 / 30.1$ & $0.015 \pm 0.0002$ & $4.55 \pm 0.01$ & $0.76 \pm 0.01$ & $2.94 \pm 1.55$ & $0.14 \pm 0.08$ & $0.0266 \pm 0.0002$ & $4.55 \pm 0.0$ & $0.77 \pm 0.01$ & $2.48 \pm 0.46$ & $0.12 \pm 0.02$ \\
\hline $3 \mathrm{C} 409$ & $63.4 /-6.1$ & $0.0058 \pm 0.0011$ & $14.59 \pm 0.27$ & $1.68 \pm 0.35$ & $11.31 \pm 8.53$ & $0.47 \pm 0.38$ & $0.0055 \pm 0.0015$ & $14.68 \pm 0.33$ & $1.52 \pm 0.4$ & $7.83 \pm 11.73$ & $0.16 \pm 0.24$ \\
\hline $3 \mathrm{C} 409$ & $63.4 /-6.1$ & $0.0204 \pm 0.0025$ & $15.4 \pm 0.01$ & $0.89 \pm 0.05$ & $3.18 \pm 2.31$ & $0.25 \pm 0.18$ & $0.0275 \pm 0.0032$ & $15.42 \pm 0.01$ & $0.86 \pm 0.04$ & $0.62 \pm 1.0$ & $0.03 \pm 0.06$ \\
\hline $3 \mathrm{C} 410$ & $69.2 /-3.8$ & $0.0044 \pm 0.0006$ & $6.32 \pm 0.04$ & $1.89 \pm 0.15$ & $13.41 \pm 11.22$ & $0.47 \pm 0.4$ & $0.0079 \pm 0.0005$ & $6.38 \pm 0.02$ & $2.32 \pm 0.09$ & $6.4 \pm 5.25$ & $0.28 \pm 0.23$ \\
\hline $3 \mathrm{C} 410$ & $69.2 /-3.8$ & $0.0089 \pm 0.0006$ & $6.21 \pm 0.01$ & $0.65 \pm 0.04$ & $8.46 \pm 2.4$ & $0.21 \pm 0.06$ & $0.0193 \pm 0.0005$ & $6.26 \pm 0.01$ & $0.81 \pm 0.02$ & $3.81 \pm 1.28$ & $0.14 \pm 0.05$ \\
\hline $3 \mathrm{C} 410$ & $69.2 /-3.8$ & $0.0044 \pm 0.0003$ & $10.7 \pm 0.03$ & $0.71 \pm 0.07$ & $10.06 \pm 5.89$ & $0.13 \pm 0.08$ & $0.0085 \pm 0.0002$ & $10.71 \pm 0.02$ & $0.81 \pm 0.04$ & $4.15 \pm 3.09$ & $0.07 \pm 0.05$ \\
\hline $3 \mathrm{C} 410$ & $69.2 /-3.8$ & $0.0054 \pm 0.0002$ & $11.67 \pm 0.03$ & $0.84 \pm 0.07$ & $4.83 \pm 4.66$ & $0.09 \pm 0.09$ & $0.0115 \pm 0.0002$ & $11.68 \pm 0.02$ & $0.82 \pm 0.03$ & $2.93 \pm 3.0$ & $0.07 \pm 0.07$ \\
\hline $3 \mathrm{C} 454.3$ & $86.1 /-38.2$ & $0.0023 \pm 0.0001$ & $-9.67 \pm 0.03$ & $1.6 \pm 0.09$ & $4.63 \pm 12.36$ & $0.07 \pm 0.19$ & $0.0044 \pm 0.0001$ & $-9.54 \pm 0.01$ & $1.25 \pm 0.04$ & $8.13 \pm 6.06$ & $0.1 \pm 0.08$ \\
\hline $3 \mathrm{C} 75$ & $170.3 /-44.9$ & $0.0071 \pm 0.0005$ & $-10.36 \pm 0.04$ & $1.3 \pm 0.12$ & $3.45 \pm 5.41$ & $0.14 \pm 0.21$ & $0.014 \pm 0.0008$ & $-10.36 \pm 0.03$ & $1.22 \pm 0.09$ & $3.51 \pm 1.56$ & $0.14 \pm 0.06$ \\
\hline $4 \mathrm{C} 13.67$ & $43.5 / 9.2$ & $0.0464 \pm 0.0043$ & $4.85 \pm 0.05$ & $1.1 \pm 0.12$ & $10.43 \pm 2.77$ & $2.28 \pm 0.69$ & $0.0567 \pm 0.0057$ & $4.89 \pm 0.05$ & $1.12 \pm 0.14$ & $10.34 \pm 2.13$ & $1.55 \pm 0.4$ \\
\hline $4 \mathrm{C} 22.12$ & $188.1 / 0.0$ & $0.0058 \pm 0.001$ & $-2.84 \pm 0.07$ & $0.79 \pm 0.19$ & $6.54 \pm 7.03$ & $0.13 \pm 0.14$ & $0.0102 \pm 0.0011$ & $-2.73 \pm 0.04$ & $0.78 \pm 0.12$ & $6.72 \pm 2.32$ & $0.13 \pm 0.05$ \\
\hline $4 C 22.12$ & $188.1 / 0.0$ & $0.0172 \pm 0.0012$ & $-1.78 \pm 0.02$ & $0.56 \pm 0.05$ & $5.07 \pm 2.12$ & $0.21 \pm 0.09$ & $0.0354 \pm 0.0013$ & $-1.78 \pm 0.01$ & $0.54 \pm 0.03$ & $3.78 \pm 0.74$ & $0.17 \pm 0.03$ \\
\hline G196.6+0.2 & $196.6 / 0.2$ & $0.0044 \pm 0.0005$ & $3.26 \pm 0.11$ & $1.94 \pm 0.27$ & $10.82 \pm 12.22$ & $0.4 \pm 0.46$ & $0.0062 \pm 0.0005$ & $3.4 \pm 0.09$ & $2.38 \pm 0.22$ & $8.85 \pm 8.6$ & $0.31 \pm 0.3$ \\
\hline G197.0+1.1 & $197.0 / 1.1$ & $0.0126 \pm 0.0005$ & $4.83 \pm 0.04$ & $1.88 \pm 0.09$ & $6.94 \pm 3.82$ & $0.7 \pm 0.39$ & $0.0191 \pm 0.001$ & $4.73 \pm 0.04$ & $1.65 \pm 0.1$ & $4.8 \pm 2.17$ & $0.36 \pm 0.16$ \\
\hline G197.0+1.1 & $197.0 / 1.1$ & $0.0059 \pm 0.0009$ & $7.46 \pm 0.05$ & $0.65 \pm 0.11$ & $0.31 \pm 10.58$ & $0.0 \pm 0.17$ & $0.0078 \pm 0.0015$ & $7.34 \pm 0.06$ & $0.65 \pm 0.14$ & $1.61 \pm 5.87$ & $0.02 \pm 0.07$ \\
\hline G197.0+1.1 & $197.0 / 1.1$ & $0.0049 \pm 0.0005$ & $17.01 \pm 0.12$ & $2.47 \pm 0.28$ & $10.99 \pm 9.9$ & $0.57 \pm 0.52$ & $0.0081 \pm 0.0034$ & $16.26 \pm 0.17$ & $0.91 \pm 0.3$ & $6.45 \pm 2.95$ & $0.11 \pm 0.08$ \\
\hline G197.0+1.1 & $197.0 / 1.1$ & $0.0052 \pm 0.0015$ & $17.59 \pm 0.03$ & $0.25 \pm 0.08$ & $9.87 \pm 1.81$ & $0.05 \pm 0.03$ & $0.0127 \pm 0.0013$ & $17.38 \pm 0.2$ & $1.46 \pm 0.35$ & $5.46 \pm 4.35$ & $0.24 \pm 0.2$ \\
\hline G197.0+1.1 & $197.0 / 1.1$ & $0.0237 \pm 0.001$ & $32.01 \pm 0.01$ & $0.57 \pm 0.03$ & $4.75 \pm 1.92$ & & $0.043 \pm 0.0017$ & & $0.54 \pm 0.02$ & $3.96 \pm 1.04$ & $0.22 \pm 0.06$ \\
\hline $\mathrm{P} 0428+20$ & $176.8 /-18.6$ & $0.0014 \pm 0.0002$ & $3.6 \pm 0.08$ & $1.01 \pm 0.19$ & $13.48 \pm 7.8$ & $0.08 \pm 0.05$ & $0.0029 \pm 0.0003$ & $3.54 \pm 0.03$ & $0.69 \pm 0.08$ & $4.45 \pm 9.98$ & $0.02 \pm 0.05$ \\
\hline $\mathrm{P} 0428+20$ & $176.8 /-18.6$ & $0.0075 \pm 0.0002$ & $10.7 \pm 0.02$ & $1.09 \pm 0.04$ & $13.49 \pm 3.45$ & $0.47 \pm 0.12$ & $0.0136 \pm 0.0002$ & $10.7 \pm 0.01$ & $1.1 \pm 0.02$ & $12.72 \pm 1.62$ & $0.45 \pm 0.06$ \\
\hline $\mathrm{T} 0526+24$ & $181.4 /-5.2$ & $0.0172 \pm 0.0073$ & $7.55 \pm 0.29$ & $1.9 \pm 1.13$ & $13.7 \pm 15.65$ & $1.91 \pm 2.59$ & $0.043 \pm 0.0102$ & $7.56 \pm 0.14$ & $2.43 \pm 0.75$ & $10.19 \pm 7.5$ & $2.52 \pm 2.1$ \\
\hline T0629+10 & $201.5 / 0.5$ & $0.0043 \pm 0.0022$ & $0.16 \pm 0.0$ & $0.65 \pm 0.4$ & $4.16 \pm 2.97$ & $0.05 \pm 0.05$ & $0.0103 \pm 0.0035$ & $0.35 \pm 0.0$ & $1.18 \pm 0.41$ & $3.25 \pm 1.93$ & $0.09 \pm 0.07$ \\
\hline T0629+10 & $201.5 / 0.5$ & $0.0387 \pm 0.0074$ & $3.14 \pm 0.13$ & $1.1 \pm 0.0$ & $3.85 \pm 0.47$ & $0.7 \pm 0.16$ & $0.0577 \pm 0.0113$ & $3.07 \pm 0.14$ & $1.1 \pm 0.0$ & $4.54 \pm 0.55$ & $0.68 \pm 0.16$ \\
\hline T0629+10 & $201.5 / 0.5$ & $0.0169 \pm 0.0015$ & $1.46 \pm 0.07$ & $1.39 \pm 0.26$ & $2.19 \pm 2.11$ & $0.22 \pm 0.22$ & $0.0281 \pm 0.0029$ & $1.51 \pm 0.08$ & $1.23 \pm 0.25$ & $2.9 \pm 0.91$ & $0.24 \pm 0.09$ \\
\hline
\end{tabular}


Table 1

(Continued)

\begin{tabular}{|c|c|c|c|c|c|c|c|c|c|c|c|}
\hline \multirow{2}{*}{$\begin{array}{l}\text { Source } \\
\text { (Name) }\end{array}$} & \multirow{2}{*}{$\begin{array}{l}l / b \\
\left({ }^{\circ}\right)\end{array}$} & \multicolumn{5}{|c|}{$\mathrm{OH}(1665)$} & \multicolumn{5}{|c|}{$\mathrm{OH}(1667)$} \\
\hline & & $\tau$ & $\begin{array}{c}V_{\mathrm{lsr}} \\
\left(\mathrm{km} \mathrm{s}^{-1}\right)\end{array}$ & $\begin{array}{c}\Delta V \\
\left(\mathrm{~km} \mathrm{~s}^{-1}\right)\end{array}$ & $\begin{array}{l}T_{\mathrm{ex}} \\
(\mathrm{K})\end{array}$ & $\begin{array}{c}N(\mathrm{OH}) \\
\left(10^{14} \mathrm{~cm}^{-2}\right)\end{array}$ & $\tau$ & $\begin{array}{c}V_{\text {lsr }} \\
\left(\mathrm{km} \mathrm{s}^{-1}\right)\end{array}$ & $\begin{array}{c}\Delta V \\
\left(\mathrm{~km} \mathrm{~s}^{-1}\right)\end{array}$ & $\begin{array}{l}T_{\text {ex }} \\
(\mathrm{K})\end{array}$ & $\begin{array}{c}N(\mathrm{OH}) \\
\left(10^{14} \mathrm{~cm}^{-2}\right)\end{array}$ \\
\hline T0629+10 & $201.5 / 0.5$ & $0.1607 \pm 0.0104$ & $3.6 \pm 0.01$ & $0.61 \pm 0.02$ & $3.83 \pm 0.35$ & $1.59 \pm 0.19$ & $0.2536 \pm 0.0154$ & $3.6 \pm 0.01$ & $0.65 \pm 0.03$ & $4.72 \pm 0.64$ & $1.84 \pm 0.29$ \\
\hline $\mathrm{T} 0629+10$ & $201.5 / 0.5$ & $0.0811 \pm 0.002$ & $4.62 \pm 0.01$ & $0.76 \pm 0.03$ & $6.43 \pm 0.97$ & $1.68 \pm 0.27$ & $0.1553 \pm 0.0037$ & $4.61 \pm 0.01$ & $0.67 \pm 0.03$ & $6.62 \pm 1.01$ & $1.63 \pm 0.26$ \\
\hline $\mathrm{T} 0629+10$ & $201.5 / 0.5$ & $0.0747 \pm 0.0018$ & $6.09 \pm 0.02$ & $1.06 \pm 0.05$ & $5.44 \pm 1.58$ & $1.84 \pm 0.54$ & $0.1165 \pm 0.003$ & $6.06 \pm 0.02$ & $1.17 \pm 0.07$ & $6.52 \pm 1.67$ & $2.1 \pm 0.55$ \\
\hline $\mathrm{T} 0629+10$ & $201.5 / 0.5$ & $0.0367 \pm 0.0031$ & $7.0 \pm 0.02$ & $0.49 \pm 0.06$ & $4.36 \pm 0.68$ & $0.33 \pm 0.07$ & $0.0631 \pm 0.0056$ & $7.0 \pm 0.02$ & $0.5 \pm 0.06$ & $4.2 \pm 0.3$ & $0.31 \pm 0.05$ \\
\hline $\mathrm{T} 0629+10$ & $201.5 / 0.5$ & $0.0174 \pm 0.0018$ & $7.9 \pm 0.05$ & $0.83 \pm 0.13$ & $3.44 \pm 1.67$ & $0.21 \pm 0.11$ & $0.0307 \pm 0.003$ & $7.91 \pm 0.05$ & $0.82 \pm 0.13$ & $3.65 \pm 1.21$ & $0.22 \pm 0.08$ \\
\hline
\end{tabular}


Table 2

34 Atomic Sightlines

\begin{tabular}{|c|c|c|c|c|c|c|c|}
\hline $\begin{array}{l}\text { Sources } \\
\text { (Name) }\end{array}$ & $\begin{array}{l}l / b \\
\left({ }^{\circ}\right)\end{array}$ & $\begin{array}{c}N_{\mathrm{H} \mathrm{I}} \\
\left(10^{20} \mathrm{~cm}^{-2}\right)\end{array}$ & $\begin{array}{c}N_{\mathrm{H}}^{*} \\
\left(10^{20} \mathrm{~cm}^{-2}\right)\end{array}$ & $\begin{array}{c}\sigma_{\tau}\left(\mathrm{OH}_{1667}\right) \\
\left(10^{-4}\right)\end{array}$ & $\begin{array}{c}N_{\mathrm{H}_{2}}(\text { upper limit })^{\mathrm{a}} \\
\quad\left(10^{20} \mathrm{~cm}^{-2}\right)\end{array}$ & $\begin{array}{c}\tau_{353} \\
\left(10^{-6}\right)\end{array}$ & $\begin{array}{c}E(B-V) \\
\left(10^{-2} \mathrm{mag}\right)\end{array}$ \\
\hline $3 \mathrm{C} 33$ & $129.4 /-49.3$ & $3.25 \pm 0.0$ & $3.2 \pm 0.1$ & 12.14 & 0.6 & $2.16 \pm 0.07$ & $3.54 \pm 0.42$ \\
\hline $3 \mathrm{C} 142.1$ & $197.6 /-14.5$ & $25.11 \pm 2.6$ & $19.6 \pm 0.8$ & 10.55 & 0.52 & $21.53 \pm 0.72$ & $21.71 \pm 0.81$ \\
\hline $3 \mathrm{C} 138$ & $187.4 /-11.3$ & $22.9 \pm 1.1$ & $19.9 \pm 0.3$ & 5.02 & 0.25 & $21.63 \pm 0.81$ & $17.47 \pm 0.59$ \\
\hline $3 \mathrm{C} 79$ & $164.1 /-34.5$ & $10.86 \pm 1.2$ & $9.8 \pm 0.8$ & 37.03 & 1.84 & $9.23 \pm 0.37$ & $12.67 \pm 0.78$ \\
\hline $3 \mathrm{C} 78$ & $174.9 /-44.5$ & $11.69 \pm 0.5$ & $10.3 \pm 0.2$ & 13.25 & 0.66 & $12.45 \pm 0.63$ & $14.64 \pm 1.07$ \\
\hline $3 \mathrm{C} 310$ & $38.5 / 60.2$ & $4.29 \pm 0.1$ & $4.0 \pm 0.1$ & 16.19 & 0.8 & $3.48 \pm 0.15$ & $2.75 \pm 0.53$ \\
\hline $3 \mathrm{C} 315$ & $39.4 / 58.3$ & $5.48 \pm 0.4$ & $4.7 \pm 0.0$ & 12.96 & 0.64 & $3.98 \pm 0.09$ & $5.63 \pm 0.26$ \\
\hline $3 \mathrm{C} 234$ & $200.2 / 52.7$ & $1.84 \pm 0.0$ & $1.9 \pm 1.1$ & 12.66 & 0.63 & $0.78 \pm 0.03$ & $1.64 \pm 0.56$ \\
\hline $3 \mathrm{C} 236$ & $190.1 / 54.0$ & $1.38 \pm 0.0$ & $1.3 \pm 1.2$ & 10.72 & 0.53 & $0.7 \pm 0.03$ & $2.04 \pm 0.49$ \\
\hline $3 \mathrm{C} 64$ & $157.8 /-48.2$ & $7.29 \pm 0.2$ & $6.9 \pm 0.8$ & 33.12 & 1.65 & $6.55 \pm 0.35$ & $9.01 \pm 0.36$ \\
\hline $\mathrm{P} 0531+19$ & $186.8 /-7.1$ & $27.33 \pm 0.7$ & $25.4 \pm 0.3$ & 6.37 & 0.32 & $20.75 \pm 0.62$ & $20.54 \pm 1.3$ \\
\hline $\mathrm{P} 0820+22$ & $201.4 / 29.7$ & $4.82 \pm 0.2$ & $4.8 \pm 1.1$ & 7.09 & 0.35 & $3.73 \pm 0.11$ & $2.56 \pm 0.4$ \\
\hline $3 \mathrm{C} 192$ & $197.9 / 26.4$ & $4.56 \pm 0.1$ & $4.5 \pm 0.1$ & 20.66 & 1.03 & $3.38 \pm 0.08$ & $4.05 \pm 0.53$ \\
\hline $3 \mathrm{C} 98$ & $179.8 /-31.0$ & $12.7 \pm 0.5$ & $11.3 \pm 1.3$ & 12.26 & 0.61 & $13.72 \pm 0.41$ & $17.73 \pm 1.02$ \\
\hline $3 \mathrm{C} 273$ & $289.9 / 64.4$ & $2.35 \pm 0.0$ & $2.3 \pm 0.1$ & 21.0 & 1.04 & $1.3 \pm 0.09$ & $1.98 \pm 0.41$ \\
\hline DW $0742+10$ & $209.8 / 16.6$ & $2.77 \pm 0.0$ & $2.8 \pm 0.9$ & 8.01 & 0.4 & $1.6 \pm 0.03$ & $1.99 \pm 0.25$ \\
\hline $3 \mathrm{C} 172.0$ & $191.2 / 13.4$ & $8.89 \pm 0.2$ & $8.6 \pm 1.1$ & 13.02 & 0.65 & $5.66 \pm 0.08$ & $4.7 \pm 0.52$ \\
\hline $3 C 293$ & $54.6 / 76.1$ & $1.46 \pm 0.1$ & $1.5 \pm 1.1$ & 6.24 & 0.31 & $1.31 \pm 0.09$ & $2.83 \pm 0.75$ \\
\hline $3 \mathrm{C} 120$ & $190.4 /-27.4$ & $18.17 \pm 2.1$ & $10.7 \pm 0.1$ & 28.29 & 1.41 & $29.26 \pm 1.08$ & $22.74 \pm 1.03$ \\
\hline CTA21 & $166.6 /-33.6$ & $10.97 \pm 0.4$ & $10.0 \pm 0.8$ & 27.35 & 1.36 & $10.39 \pm 0.44$ & $13.3 \pm 0.96$ \\
\hline $\mathrm{P} 1117+14$ & $240.4 / 65.8$ & $1.79 \pm 0.0$ & $1.8 \pm 0.3$ & 15.0 & 0.75 & $1.5 \pm 0.04$ & $2.73 \pm 0.54$ \\
\hline $3 \mathrm{C} 264.0$ & $237.0 / 73.6$ & $1.97 \pm 0.0$ & $2.0 \pm 0.4$ & 6.29 & 0.31 & $1.64 \pm 0.08$ & $2.88 \pm 0.34$ \\
\hline 3C208.1 & $213.6 / 33.6$ & $3.15 \pm 0.1$ & $3.2 \pm 0.2$ & 18.67 & 0.93 & $3.12 \pm 0.04$ & $2.93 \pm 0.43$ \\
\hline $3 \mathrm{C} 208.0$ & $213.7 / 33.2$ & $3.41 \pm 0.1$ & $3.5 \pm 0.2$ & 19.69 & 0.98 & $3.38 \pm 0.07$ & $4.37 \pm 0.47$ \\
\hline $4 C 32.44$ & $67.2 / 81.0$ & $1.23 \pm 0.0$ & $1.3 \pm 0.6$ & 9.88 & 0.49 & $0.81 \pm 0.02$ & $1.94 \pm 0.29$ \\
\hline $3 \mathrm{C} 272.1$ & $280.6 / 74.7$ & $2.82 \pm 0.0$ & $2.8 \pm 0.3$ & 10.24 & 0.51 & $1.73 \pm 0.28$ & $2.43 \pm 0.31$ \\
\hline $4 \mathrm{C} 07.32$ & $322.2 / 68.8$ & $2.43 \pm 0.0$ & $2.4 \pm 0.3$ & 30.7 & 1.53 & $2.32 \pm 0.06$ & $4.3 \pm 0.41$ \\
\hline $3 \mathrm{C} 245$ & $233.1 / 56.3$ & $2.39 \pm 0.0$ & $2.4 \pm 0.2$ & 11.36 & 0.56 & $2.22 \pm 0.06$ & $2.71 \pm 0.36$ \\
\hline $3 \mathrm{C} 348$ & $23.0 / 29.2$ & $6.56 \pm 0.2$ & $6.0 \pm 0.1$ & 16.51 & 0.82 & $5.7 \pm 0.15$ & $9.8 \pm 0.33$ \\
\hline $3 \mathrm{C} 286$ & $56.5 / 80.7$ & $2.33 \pm 0.1$ & $2.4 \pm 2.7$ & 7.13 & 0.35 & $0.81 \pm 0.05$ & $2.75 \pm 0.74$ \\
\hline $4 C 13.65$ & $39.3 / 17.7$ & $10.56 \pm 0.2$ & $9.9 \pm 0.1$ & 20.01 & 0.99 & $11.8 \pm 0.39$ & $15.63 \pm 0.6$ \\
\hline $3 \mathrm{C} 190.0$ & $207.6 / 21.8$ & $3.21 \pm 0.0$ & $3.4 \pm 0.9$ & 17.57 & 0.87 & $2.41 \pm 0.03$ & $1.96 \pm 0.42$ \\
\hline $3 \mathrm{C} 274.1$ & $269.9 / 83.2$ & $2.74 \pm 0.0$ & $2.6 \pm 0.1$ & 13.13 & 0.65 & $2.34 \pm 0.02$ & $2.64 \pm 0.42$ \\
\hline $3 C 298$ & $352.2 / 60.7$ & $2.39 \pm 0.4$ & $2.6 \pm 0.1$ & 10.69 & 0.53 & $1.3 \pm 0.07$ & $1.97 \pm 0.39$ \\
\hline
\end{tabular}

Note.

${ }^{\text {a }}$ Estimated from $\mathrm{OH}(1667) 3 \sigma$ detection limits using $T_{\mathrm{ex}}=3.5 \mathrm{~K}, \mathrm{FWHM}=1 \mathrm{~km} \mathrm{~s}^{-1}$ and $N_{\mathrm{OH}} / N_{\mathrm{H}_{2}}=10^{-7}$ (see Section 5).

\section{1. $\mathrm{N}_{H}$ from Dust Optical Depth $\tau_{353}$}

We adopt the all-sky map of dust optical depth $\tau_{353}$ computed by PLC2014a. This was derived from an MBB empirical fit to IRAS and Planck maps at 3000, 857, 545, and $353 \mathrm{GHz}$, described by the expression:

$$
I_{\nu}=\tau_{353} B_{\nu}\left(T_{\text {dust }}\right)\left(\frac{\nu}{353}\right)^{\beta_{\text {dust }}} .
$$

Here, $\tau_{353}$, dust temperature, $T_{\text {dust }}$, and spectral index, $\beta_{\text {dust }}$, are the three free parameters, and $B_{\nu}\left(T_{\text {dust }}\right)$ is the Planck function for dust at temperature $T_{\text {dust }}$ which is, in this model, considered to be uniform along each sightline (see PLC2014a for more details). The relation between dust optical depth and total gas column density can then be written as:

$$
\tau_{353}=\frac{I_{353}}{B_{353}\left(T_{\text {dust }}\right)}=\kappa_{353} r \mu m_{\mathrm{H}} N_{\mathrm{H}}=\sigma_{353} N_{\mathrm{H}},
$$

where $\sigma_{353}$ is the dust opacity, $\kappa_{353}$ is the dust emissivity crosssection per unit mass $\left(\mathrm{cm}^{2} \mathrm{~g}^{-1}\right), r$ is the dust-to-gas mass ratio, $\mu$ is the mean molecular weight, and $m_{\mathrm{H}}$ is the mass of a hydrogen atom.

Figure 7 shows the correlation between $N_{\mathrm{H}}$ and $\tau_{353}$. A tight linear trend can be seen with a Pearson coefficient of 0.95 . The value of $\sigma_{353}$ from the orthogonal distance regression (Boggs \& Rogers 1990) linear fit is $(7.9 \pm 0.6) \times 10^{-27} \mathrm{~cm}^{2} \mathrm{H}^{-1}$ (the intercept is set to 0 ), where the quoted uncertainties are the 95\% confidence limits estimated from pair bootstrap resampling. This is consistent to within the uncertainties with that obtained by PLC2014a based on all-sky H I data from LAB, $(6.6 \pm 1.7) \times 10^{-27} \mathrm{~cm}^{2} \mathrm{H}^{-1}$. Note that here we have quoted the PLC2014a measurement made toward low $N_{\mathrm{H} \text { I }}$ positions, because the lack of any $\mathrm{H}$ I opacity correction in that work makes this value the most reliable. However, our fit is consistent with all of the $\sigma_{353}$ values presented in that work (which was based on the Planck R1.20 data release), to within the quoted uncertainties.

Small systematic deviations from the linear fit, evident at the high and low column density ends of the plot, are discussed further in Section 4.3. 

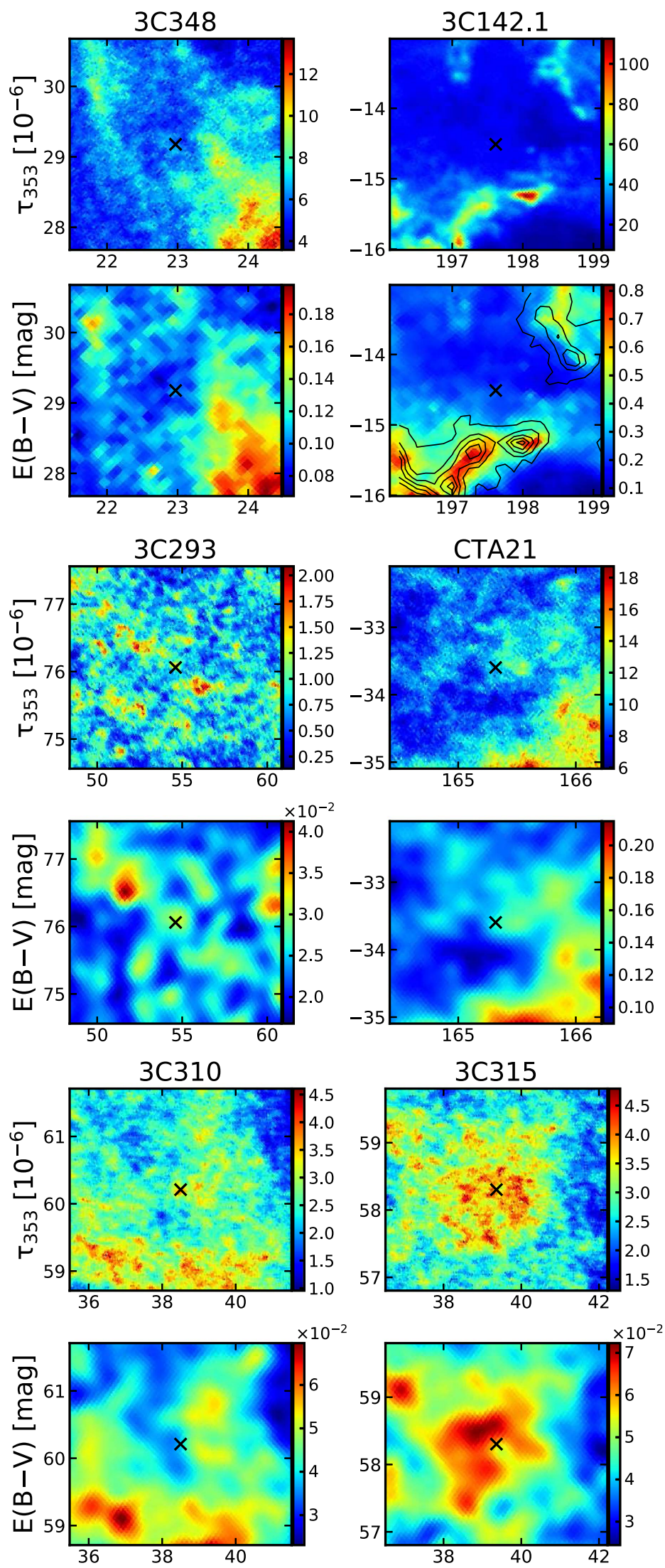

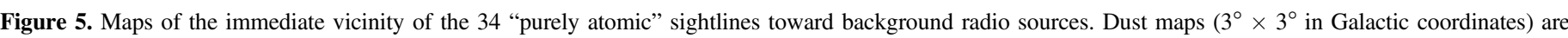

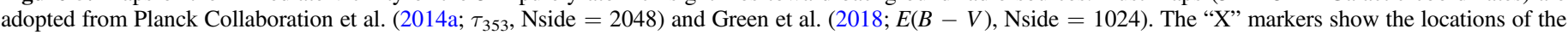

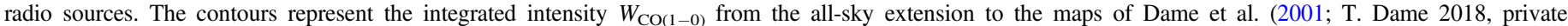

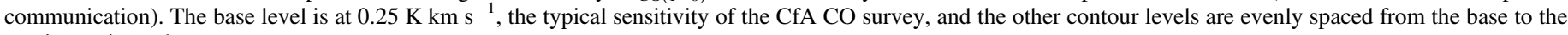
maximum in each map area. 

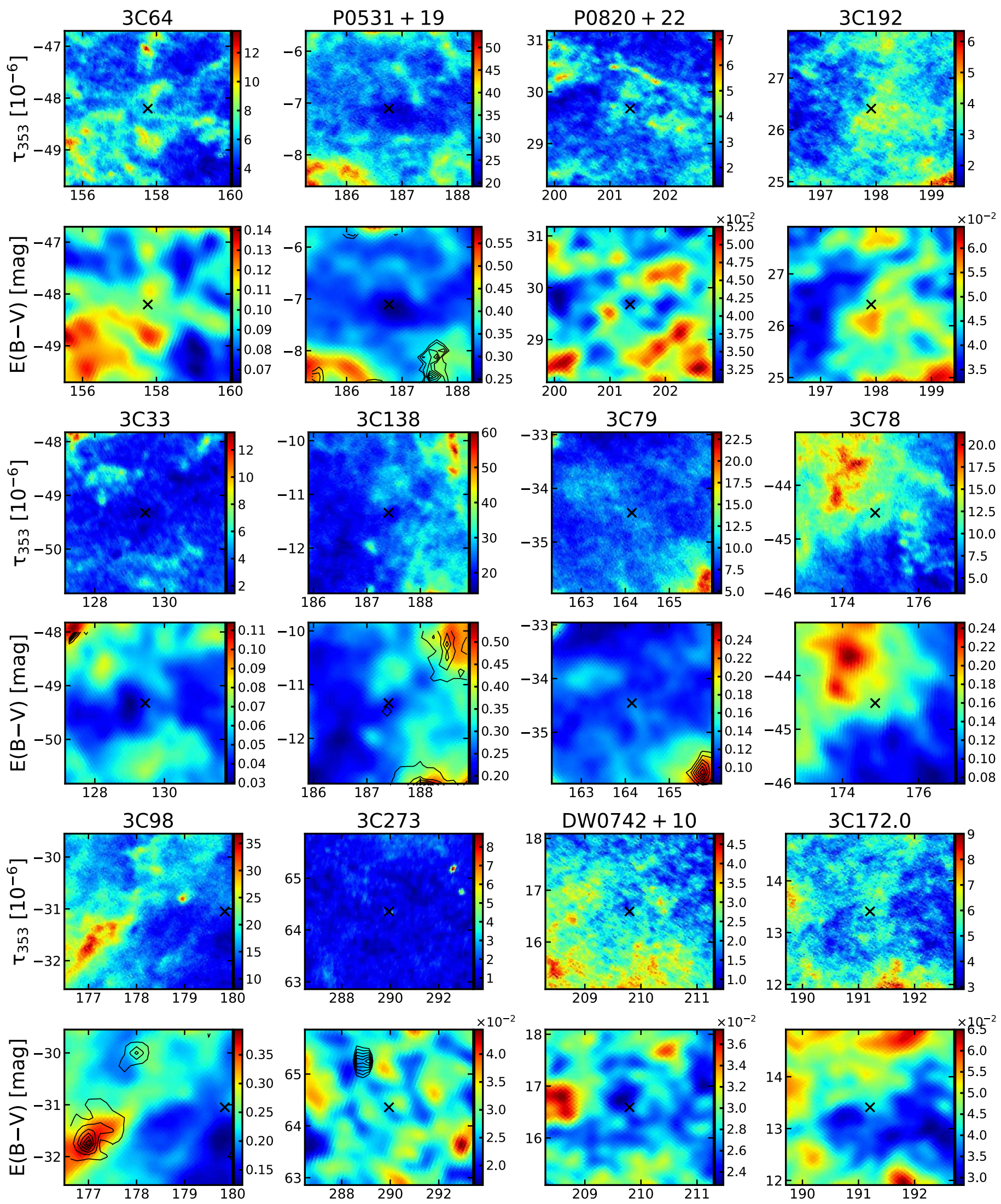

Figure 5. (Continued.)

In order to examine the possible contribution of molecular gas to $N_{\mathrm{H}}$ along the 34 atomic sightlines, we estimate upper limits on $N_{\mathrm{H}_{2}}$ from the $3 \sigma \mathrm{OH}$ detection limits using an abundance ratio of $X_{\mathrm{OH}}=10^{-7}$ (see Section 5). These values are tabulated in Table 2 , and the resulting upper limits on $N_{\mathrm{H}}$ are shown as gray triangles in Figure 7. As 

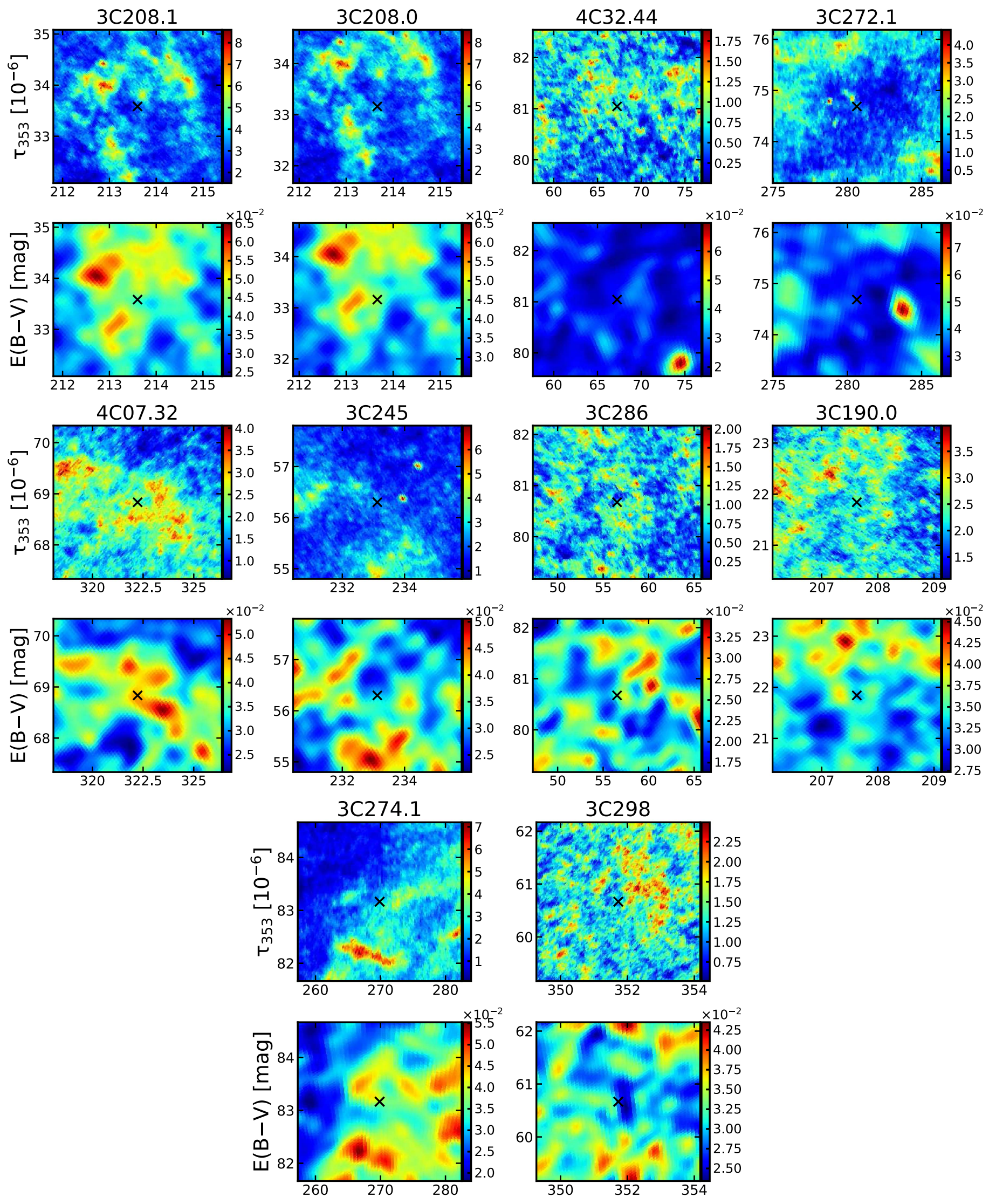

Figure 5. (Continued.) 

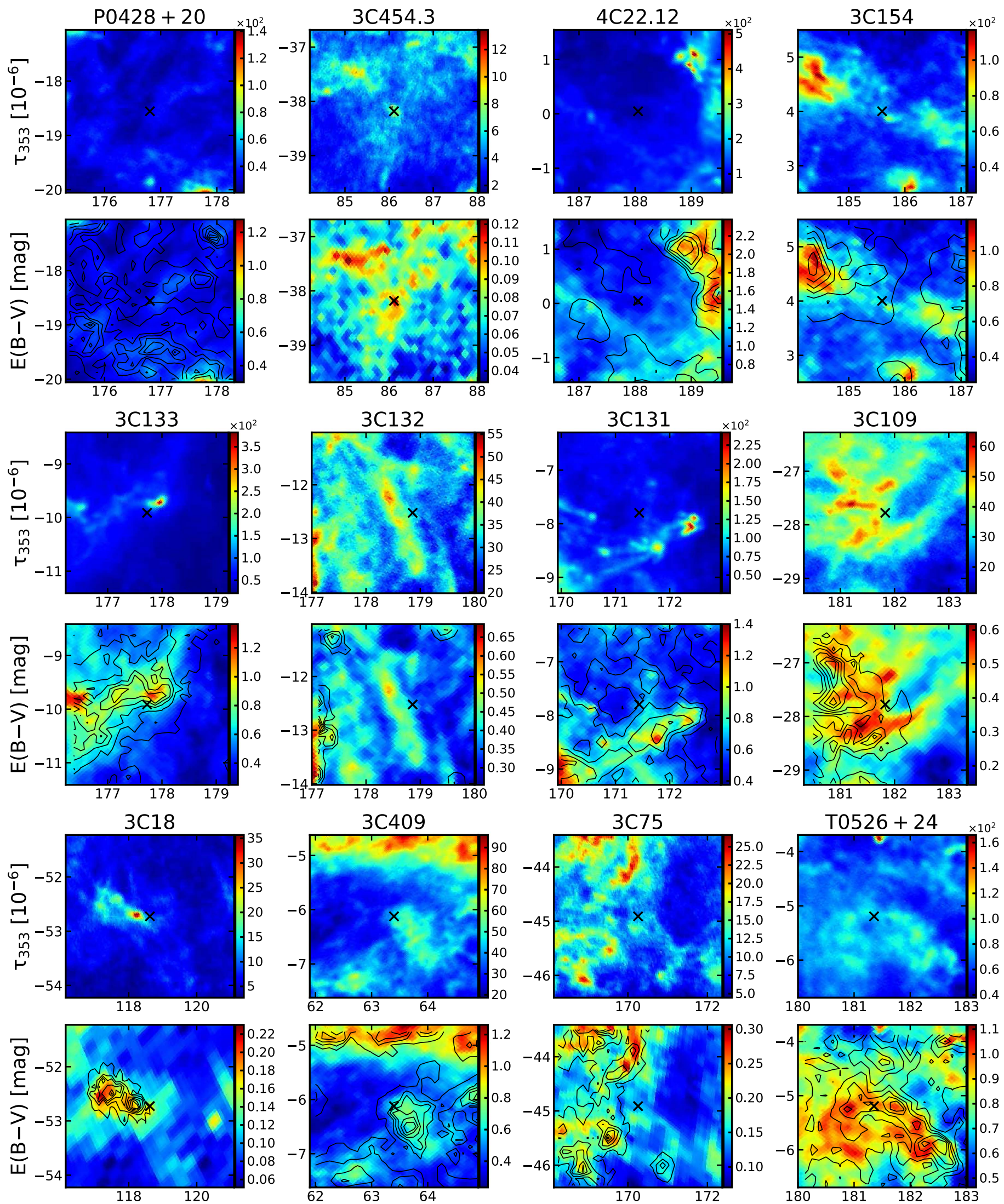

Figure 6. Maps of the immediate vicinity of the $19 \mathrm{OH}$-detected sightlines toward background radio sources. Dust maps $\left(3^{\circ} \times 3^{\circ}\right.$ in Galactic coordinates) are adopted from Planck Collaboration et al. $\left(2014 \mathrm{a} ; \tau_{353}\right.$, Nside $\left.=2048\right)$ and Green et al. $(2018 ; E(B-V)$, Nside $=1024)$. The "X" markers show the locations of the radio sources. The contours represent the integrated intensity $W_{\mathrm{CO}(1-0)}$ from the all-sky extension to the maps of Dame et al. (2001; T. Dame 2018, private communication). The base level is at $0.25 \mathrm{~K} \mathrm{~km} \mathrm{~s}^{-1}$, the typical sensitivity of the CfA CO survey, and the other contour levels are evenly spaced from the base to the maximum in each map area. 

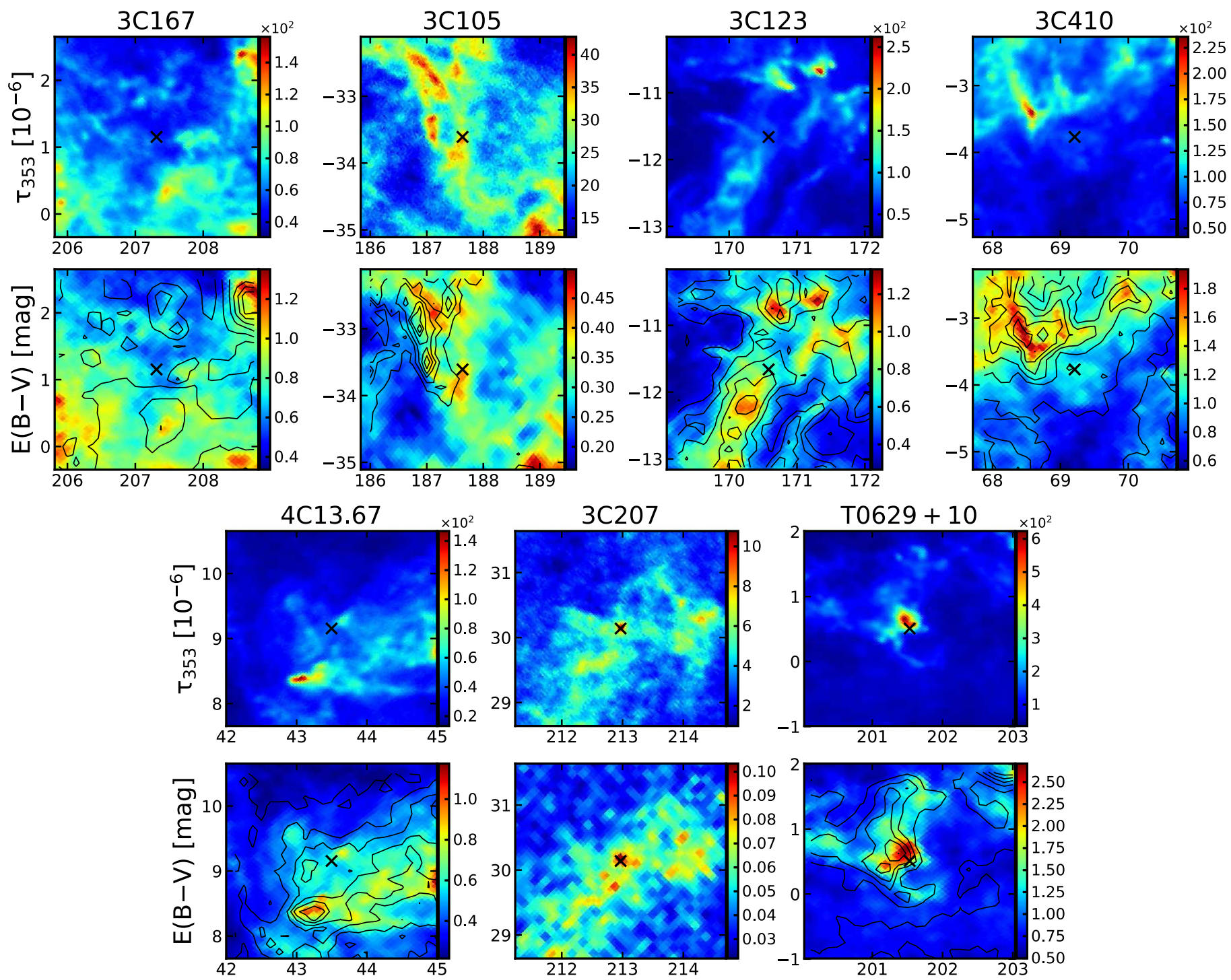

Figure 6. (Continued.)

expected, the $\sigma_{353}$ obtained from the fit to these upper limits is lower, at $(6.4 \pm 0.3) \times 10^{-27} \mathrm{~cm}^{2} \mathrm{H}^{-1}$. However, while some molecular gas may indeed be present at low levels, these limits should be considered as extreme upper bounds on the true molecular column density. This is particularly true for the most diffuse sightlines with the lowest column density $\left(N_{\mathrm{H} \mathrm{I}}<5 \times 10^{20} \mathrm{~cm}^{-2}\right)$, where the observational upper limits may appear to raise $N_{\mathrm{H}}$ by up to $\sim 50 \%$. Molecules are not expected to be well-shielded at such low columns (and indeed even CNM is largely absent along these sightlines in our data). Even for higher column density data points, it can be readily seen from Figures 5 that all sightlines considered in this analysis lie well away from even the faintest outskirts of CO-bright molecular gas complexes. We also note that the deviations from the linear fit that will be discussed in more detail below could not be removed by any selective addition of molecular gas at levels up to these limits.

We next compare our results with the dust opacity $\sigma_{353}$ derived by Fukui et al. (2015) (plotted on Figure 7 as a dashed line). These authors derived a smaller value than in

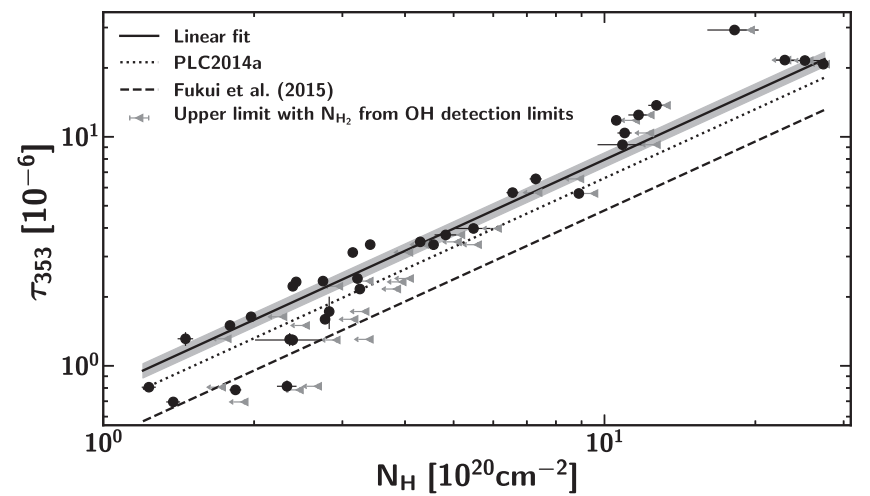

Figure 7. $\tau_{353}$ vs. $N_{\mathrm{H}}$ along the 34 purely atomic sightlines described in the text. Gray triangles indicate the upper limits for $N_{\mathrm{H}}$ along these 34 atomic sightlines with $N_{\mathrm{H}_{2}}$ estimated from the $3 \sigma \mathrm{OH}$ detection limits using an abundance ratio $N_{\mathrm{OH}} / N_{\mathrm{H}_{2}}=10^{-7}$. The thick solid line shows the linear fit to the data in this work, the dotted line shows the conversion factor derived by PLC2014a, and the dashed line shows the conversion factor derived by Fukui et al. (2015). (Note that all these works use the same $\tau_{353}$ map). $\tau_{353}$ error bars are from the uncertainty map of PLC2014a; the shaded region represents the $95 \%$ confidence intervals for the linear fit, estimated from pair bootstrap resampling. 


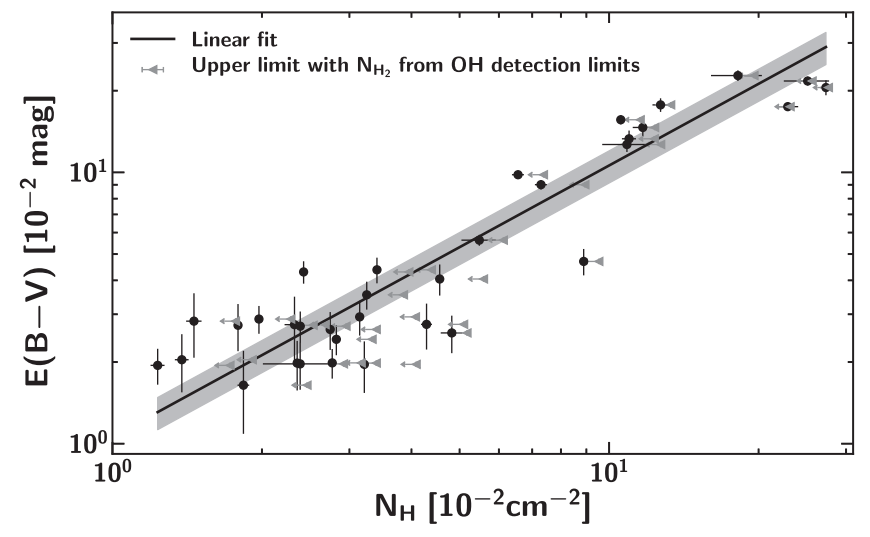

Figure 8. Correlation between $N_{\mathrm{H}}$ and dust reddening $E(B-V)$ from Green et al. (2018) along 34 atomic sightlines. Gray triangles indicate the upper limits for $N_{\mathrm{H}}$ along these 34 atomic sightlines, with $N_{\mathrm{H}_{2}}$ estimated from the $3 \sigma \mathrm{OH}$ detection limits using an abundance ratio $N_{\mathrm{OH}} / N_{\mathrm{H}_{2}}=10^{-7}$. The errorbar on $E(B-V)$ along each sightline is the standard deviation of the 20 Markov Chain realizations of $E(B-V)$ at infinite distance; the shaded region represents the $95 \%$ confidence intervals for the linear fit, estimated from pair bootstrap resampling.

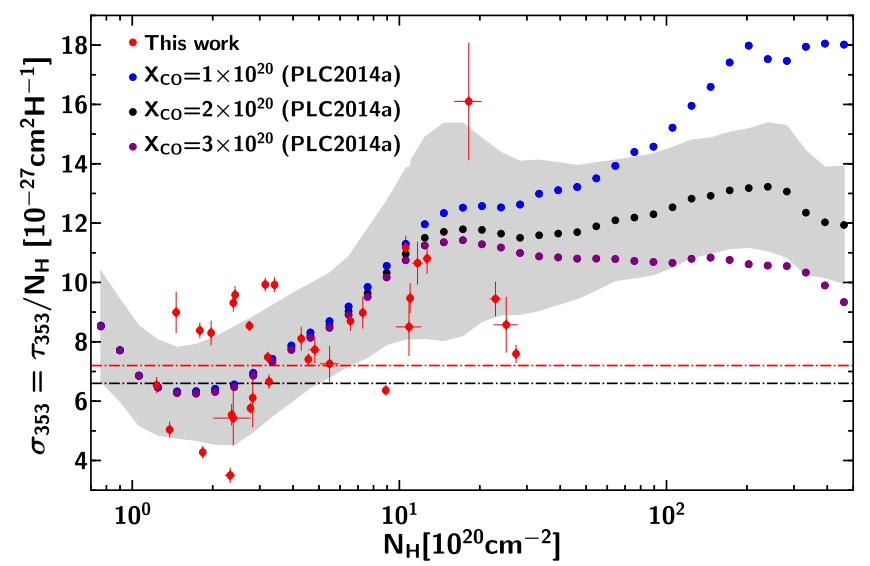

Figure 9. Dust opacity $\sigma_{353}$ vs. total column density $N_{\mathrm{H}}$ along the 34 purely atomic sightlines presented in this work (red points), overlaid on $\sigma_{353}$ derived for the whole sky at 30' resolution from PLC2014a. Here, blue points assume an X-factor of $X_{\mathrm{CO}}=1.0 \times 10^{20}$, black assume $X_{\mathrm{CO}}=2.0 \times 10^{20}$, and violet assume $X_{\mathrm{CO}}=3.0 \times 10^{20}$. The gray envelope is the standard deviation of these all-sky measurements for $X_{\mathrm{CO}}=2.0 \times 10^{20}$. The red and black dashed lines show, respectively, the constant $\sigma_{353}$ derived from the linear fit in Section 4.1 and that obtained from PLC2014a for the low column density regime.

the present work (by a factor of $\sim 1.5$ ), by restricting their fit to only the warmest dust temperatures, under the assumption that these most reliably select for genuinely optically thin H I. They then applied this factor to the Planck $\tau_{353}$ map (excluding $|b|<15^{\circ}$ and CO-bright sightlines) to estimate $N_{\mathrm{H} \text { I }}$, assuming that the contribution from $\mathrm{CO}$-dark $\mathrm{H}_{2}$ was negligible. This resulted in $N_{\mathrm{H} \text { I }}$ values $\sim 2-2.5$ times higher than under the optically thin assumption, and motivated their hypothesis that significantly more optically thick H I exists than is usually assumed. However, we find that while the $\sigma_{353}$ of Fukui et al. (2015) may be a good fit to some sightlines in the very low $N_{\mathrm{H} \text { I }}$ regime $\left(\lesssim 3 \times 10^{20} \mathrm{~cm}^{-2}\right)$, it overestimates $N_{\mathrm{H} \text { I }}$ at larger column densities by $\sim 50 \%$. Indeed, as will be discussed below, $\sigma_{353}$ is not expected to remain constant as dust evolves. This (combined with some

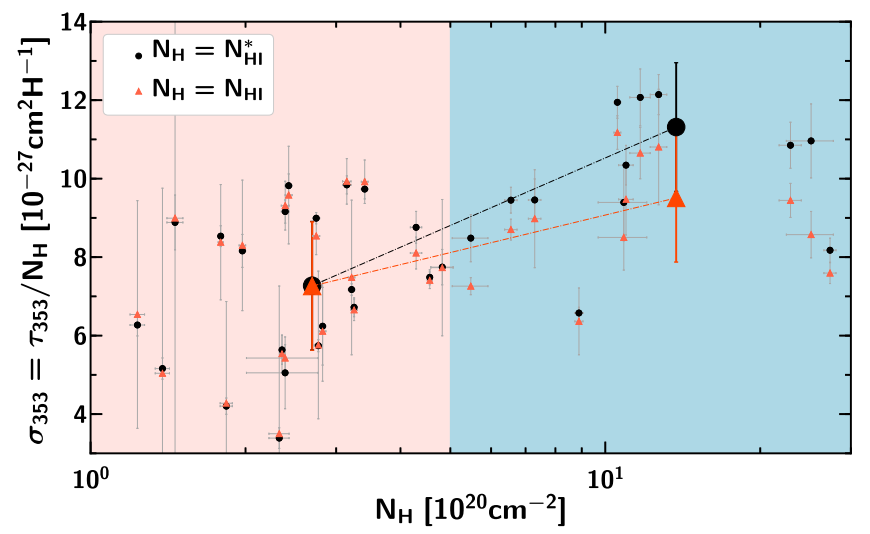

Figure 10. Dust opacity $\sigma_{353}$ vs. total column density $N_{\mathrm{H}}$ along the 34 purely atomic sightlines presented in this work using true $N_{\mathrm{H} \text { I }}$ (red) and $N_{\mathrm{H}}^{*}$ (black) as the total gas column density $N_{\mathrm{H}}$. The large data points are the average values for the low-density $\left(N_{\mathrm{H}}<5 \times 10^{20} \mathrm{~cm}^{-2}\right)$ and high-density $\left(N_{\mathrm{H}}>5 \times\right.$ $10^{20} \mathrm{~cm}^{-2}$ ) regions (error bars on these points are the standard error of the mean). Note that two data points, one black $\left(\sigma_{353}=27.2 \times 10^{-27} \mathrm{~cm}^{2} \mathrm{H}^{-1}\right)$ and one red $\left(\sigma_{353}=16.1 \times 10^{-27} \mathrm{~cm}^{2} \mathrm{H}^{-1}\right)$, at $N_{\mathrm{H}}=18.2 \times 10^{20} \mathrm{~cm}^{-2}$ are not shown, but are included in the averages.

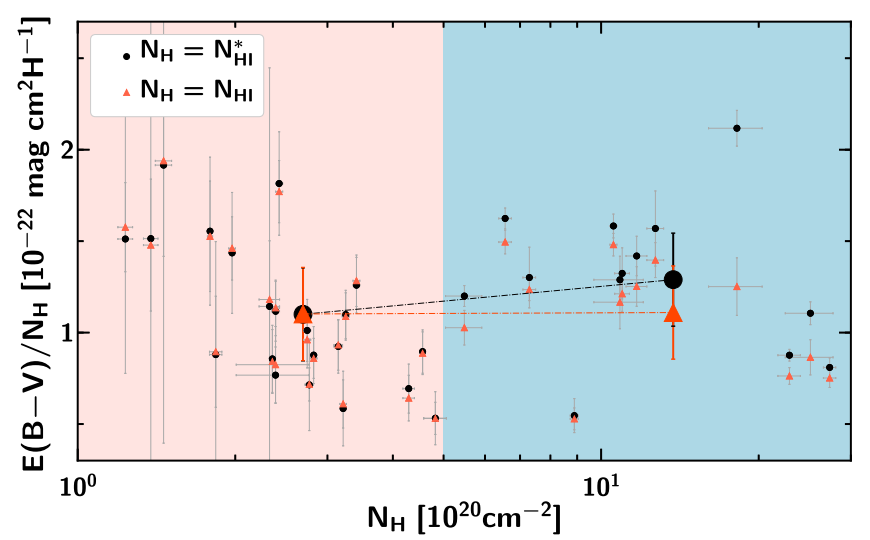

Figure 11. Ratio $E(B-V) / N_{\mathrm{H}}$ as a function of $N_{\mathrm{H}}$ along the 34 purely atomic sightlines presented in this work, using true $N_{\mathrm{H} \text { I }}$ (red) and $N_{\mathrm{H} \text { I }}^{*}$ (black) as the total gas column density $N_{\mathrm{H}}$. The large data points are the average values for the low-density $\left(N_{\mathrm{H}}<5 \times 10^{20} \mathrm{~cm}^{-2}\right)$ and high-density $\left(N_{\mathrm{H}}>5 \times 10^{20} \mathrm{~cm}^{-2}\right)$ regions (error bars for these points are the standard error on the mean).

contribution from $\mathrm{CO}$-dark $\mathrm{H}_{2}$ ) may reconcile the apparent discrepancy between their findings and absorption/emissionbased measurements of the opacity-corrected H I column.

\section{2. $\mathrm{N}_{H}$ from Dust Reddening $\mathrm{E}(\mathrm{B}-\mathrm{V})$}

Reddening caused by the absorption and scattering of light by dust grains is defined as:

$$
E(B-V)=\frac{A_{V}}{R_{V}}=1.086 \frac{\kappa_{V}}{R_{V}} r \mu m_{\mathrm{H}} N_{\mathrm{H}},
$$

where $A_{V}$ is the dust extinction, $R_{V}$ is an empirical coefficient correlated with the average grain size, and all other symbols are defined as before. In the Milky Way, $R_{V}$ is typically assumed to be 3.1 (Schultz \& Wiemer 1975), but it may vary between 2.5 and 6.0 along different sightlines (Goodman et al. 1995; Draine 2003). 

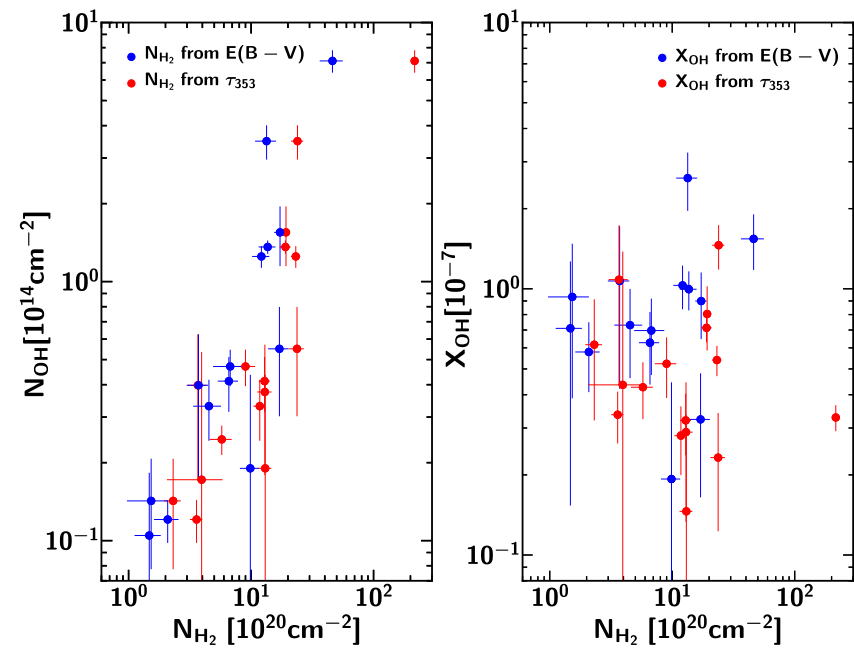

Figure 12. Left: $N_{\mathrm{OH}}$ as a function of $N_{\mathrm{H}_{2}}$ obtained from the two $N_{\mathrm{H}}$ proxies, $E$ $(B-V)$ (blue) and $\tau_{353}$ (red). Right: $X_{\mathrm{OH}}$ derived from the two proxies as a function of $\mathrm{N}_{\mathrm{H}_{2}}$.

The ratio $\left\langle N_{\mathrm{H}} / E(B-V)\right\rangle=5.8 \times 10^{21} \mathrm{~cm}^{-2} \mathrm{mag}^{-1}$ (Bohlin et al. 1978) is a widely accepted standard, used in many fields of astrophysics to connect reddening measurements to gas column density. This value was derived from $\mathrm{Ly} \alpha$ and $\mathrm{H}_{2}$ line absorption measurements toward 100 stars (see also Savage et al. 1977), and has been replicated over the years via similar methodology (e.g., Shull \& van Steenberg 1985; Diplas \& Savage 1994; Rachford et al. 2009). However, a number of recent works using $\mathrm{HI} 21 \mathrm{~cm}$ data have found significantly higher values (PLC2014a; Liszt 2014a; Lenz et al. 2017).

Here we use the all-sky map of $E(B-V)$ from Green et al. (2018) to estimate the ratio $N_{\mathrm{H}} / E(B-V)$ for our sample of purely atomic sightlines, at $|b|>5^{\circ}$. The results are shown in Figure 8. It can be seen that $E(B-V)$ and $N_{\mathrm{H}}$ are strongly linearly correlated, with a Pearson coefficient of 0.93. The ratio obtained from the linear fit is $N_{\mathrm{H}} / E(B-V)=(9.4 \pm 1.6) \times 10^{21} \mathrm{~cm}^{-2} \mathrm{mag}^{-1}$ (the intercept is also set to be 0 ), where the quoted uncertainties are the $95 \%$ confidence limits estimated from pair bootstrap resampling. This value is a factor of 1.6 higher than that in Bohlin et al. (1978).

The value obtained here is consistent with the estimate of Lenz et al. (2017): $N_{\mathrm{H}} / E(B-V)=8.8 \times 10^{21} \mathrm{~cm}^{-2} \mathrm{mag}^{-1}$ (no uncertainty is given in that work). These authors compared optically thin H I column density from HI4PI (Collaboration et al. 2016) with various estimates of $E(B-V)$ from Schlegel et al. (1998), Peek \& Graves (2010), Schlafly et al. (2014), PLC2014a, and Meisner \& Finkbeiner (2015). We note that the estimate of Lenz et al. (2017) is only valid for $N_{\mathrm{H}}<4 \times 10^{20} \mathrm{~cm}^{-2}$, where it seems safe to assume that the $21 \mathrm{~cm}$ emission is optically thin. Our value is also close to that of Liszt (2014a), who find $N_{\mathrm{H} \mathrm{I}} / E(B-V)=8.3 \times 10^{21} \mathrm{~cm}^{-2} \mathrm{mag}^{-1}$ (also given without uncertainty) for $|b| \geqslant 20^{\circ}$ and $0.015 \lesssim E(B-V) \lesssim 0.075$, by comparing $\mathrm{H}$ I data from LAB and $E(B-V)$ from Schlegel et al. (1998). The methodology used by these two studies differs in a number of details. For instance, Liszt (2014a) did not apply a gain correction to the Schlegel et al. (1998) map (whereas Lenz et al. 2017 scaled it down by 12\%), and did not smooth it to the LAB angular resolution (30'). However, Liszt (2014a) did apply an empirical correction factor to account for H I opacity (albeit one whose effects on high-latitude sightlines was small). These details may account for the difference between the values obtained by these two otherwise similar studies.

We also note that, like the present work, these studies did not take into account the potential contribution of dust associated with the diffuse warm ionized gas (WIM). This would tend to produce a flattening of the $E(B-V)$ versus $N_{\mathrm{H} \text { I }}$ relation at low $N_{\mathrm{H} \text { I }}$ and therefore increase the value of $N_{\mathrm{H} \mathrm{I}} / E(B-V)$ artificially. Because we are able to accurately probe a large column density range (up to $3 \times 10^{21} \mathrm{~cm}^{-2}$ ), we would naively expect our estimate of $N_{\mathrm{H}} / E(B-V)$ to be less affected by WIM bias than either Liszt (2014a) or Lenz et al. (2017; which would tend to have a greater effect on lower column data points). While more work is needed to quantify the contribution of the WIM on dust emission/absorption measurements at low $E(B-V)$, we consider it unlikely to account for the difference between our work and historically lower measurements of the $N_{\mathrm{H}} / E(B-V)$ ratio.

Despite minor differences between these three studies, it is clear that they point to a $N_{\mathrm{H} \mathrm{I}} / E(B-V)$ value of $(\sim 8-9) \times 10^{21} \mathrm{~cm}^{-2} \mathrm{mag}^{-1}$. This is $40 \%-60 \%$ higher than the traditional value of Bohlin et al. (1978), which has been used by most models of interstellar dust as a reference point to set the dust-to-gas ratio (e.g., Draine \& Fraisse 2009; Jones et al. 2013). We note that if $N_{\mathrm{H}}$ is replaced with upper limits (as discussed in Section 4.1), $N_{\mathrm{H}} / E(B-V)$ climbs yet higher, leaving this key conclusion unaffected.

\subsection{Disentangling the Effects of Grain Evolution and Dark Gas on $\sigma_{353}$}

A number of studies have used the correlation between $\tau_{353}$ and $N_{\mathrm{H}}$, particularly with regards to the search for dark gas (e.g., Planck Collaboration et al. 2011; Fukui et al. 2014, 2015; Reach et al. 2015). It is clear that $\tau_{353}$ and $N_{\mathrm{H}}$ are in general linearly correlated only if $\sigma_{353}$ is a constant. However, it is recognized that $\sigma_{353}$ is sensitive to grain evolution, and significant variations in the ratio $N_{\mathrm{H}} / \tau_{353}$ have been observed, particularly when transitioning to the high-density, molecular regime (e.g., Planck Collaboration et al. 2014a, 2015; Okamoto et al. 2017; Remy et al. 2017). The origin of observed variations in $\sigma_{353}$ may relate to a change in dust properties via $\kappa_{353}$, and/or a variation in the dust-to-gas ratio $r$, but may also include a contribution due to the presence of dark gas, if this is unaccounted for in the estimated $N_{\mathrm{H}}$.

PLC2014a presented the variation in $\sigma_{353}$ with $N_{\mathrm{H}}$ at $30^{\prime}$ resolution over the entire sky. In that work, $N_{\mathrm{H}}$ was derived from $\left(N_{\mathrm{H}}^{*}+X_{\mathrm{CO}} \mathrm{W}_{\mathrm{CO}}\right)$, thus dark gas (both optically thick $\mathrm{H} \mathrm{I}$ and $\mathrm{CO}$-dark $\mathrm{H}_{2}$ ) was unaccounted for. We reproduce their data in Figure 9. It can be seen that $\sigma_{353}$ is roughly flat and at a minimum in a narrow, low column density range $N_{\mathrm{H}}=$ $(1-3) \times 10^{20} \mathrm{~cm}^{-2}$, then increases linearly until $N_{\mathrm{H}}=15 \times$ $10^{20} \mathrm{~cm}^{-2}$, by which point it is almost a factor of 2 higher. It then remains approximately constant for the canonical value of $X_{\mathrm{CO}}=2.0 \times 10^{20} \mathrm{~cm}^{-2} \mathrm{~K}^{-1} \mathrm{~km}^{-1} \mathrm{~s}$. A key issue for dark gas studies is disentangling how much of the initial rise in $\sigma_{353}$ is due to changing grain properties and how much is due to the contribution of unseen material, whether it be opaque $\mathrm{HI}$ or diffuse $\mathrm{H}_{2}$. (Note also the upturn in $\sigma_{353}$ seen at the lowest $N_{\mathrm{H}}$, which may be due to the presence of unaccounted-for protons in the warm ionized medium.)

The column density range probed by our purely atomic sightlines, $N_{\mathrm{H}}=(1 \sim 30) \times 10^{20} \mathrm{~cm}^{-2}$ well samples the range where $\sigma_{353}$ undergoes its first linear increase. Dark gas is also 
fully accounted for in our data, since H I is opacity-corrected, and no molecular gas is detected in emission along these sightlines. To quantify the effect of ignoring H I opacity on $\sigma_{353}$, we compare $\sigma_{353}$ deduced from the true, opacitycorrected $N_{\mathrm{H} \text { I }}$ with that deduced under the optically thin assumption. The results are shown in Figure 10. In low column density regions $\left(N_{\mathrm{H}}<5 \times 10^{20} \mathrm{~cm}^{-2}\right)$, each $\sigma_{353}$ pair from $N_{\mathrm{H} \text { I }}$ and $N_{\mathrm{H} \text { I }}^{*}$ are comparable. However, at higher column densities $\left(N_{\mathrm{H}}>5 \times 10^{20} \mathrm{~cm}^{-2}\right) \sigma_{353}$ from true $N_{\mathrm{H} \mathrm{I}}$ is systematically lower than that measured from $N_{\mathrm{H} \text { I }}^{*}$. On average, $\sigma_{353}$ obtained from optically thin H I column density increases by $\sim 1.6$ when going from low to high column density regions; whereas $\sigma_{353}$ from true $N_{\mathrm{H} \text { I }}$ increases by $\sim 1.4$. This suggests that if $\mathrm{H} \mathrm{I}$ opacity is not explicitly corrected for, it can account for around one-third (1/3) of the increase of $\sigma_{353}$ observed during the transition from diffuse to dense atomic regimes. The remaining of two-thirds $(2 / 3)$ must arise due to changes in dust properties.

From Equation (11), we see that $\sigma_{353}$ is a function of the dust-to-gas mass ratio, $r$, and the dust emissivity cross-section, $\kappa_{353}$, which depends on the composition and structure of dust grains. Given the uncertainties on the efficiency of the physical processes involved in the evolution of interstellar dust grains, it is difficult at this point to conclude if the variations of $\sigma_{353}$ observed here are due to an increase of the dust mass (i.e., $r$ ) or to a change in the dust emission properties (i.e., $\kappa_{353}$ ). Using the dust model of Jones et al. (2013), Ysard et al. (2015) suggest that most of the variations in the dust emission observed by Planck in the diffuse ISM could be explained by relatively small variations in the dust properties. That interpretation would favor a scenario in which the increase of $\sigma_{353}$ from diffuse to denser gas is caused by the growth of thin mantles via the accretion of atoms and molecules from the gas phase. Even though this process would increase the mass of grains (and therefore increase $r$ ), the change of the structure of the grain surface would lead to a larger increase in $\kappa_{353}$. Alternatively, it is possible that this systematic variation of $\tau_{353} / N_{\mathrm{H}}$ could be due to residual large-scale systematic effects in the Planck data, or to the fact that the modified blackbody model introduces a bias in the estimate of $\tau_{353}$. Neither of these explanations can be ruled out.

Figure 9 shows $\sigma_{353}$ as a function of $N_{\mathrm{H}}$ superimposed on the results from PLC2014a. It can be seen that we observe a similar rise in $\sigma_{353}$ in the column density range $(\sim 5-30) \times 10^{20} \mathrm{~cm}^{-2}$, but less extreme. In particular, most of our data points in the higher column density range $\left(N_{\mathrm{H}}>5 \times 10^{20} \mathrm{~cm}^{-2}\right)$ are found below the PLC2014a trend, which is derived from the mean values of $\sigma_{353}$ over the whole sky in $N_{\mathrm{H}}$ bins. This is true even if we use $N_{\mathrm{H} \text { I }}^{*}$ rather than $N_{\mathrm{H} \text { I }}$ to derive $\sigma_{353}$, indicating that optically thick $\mathrm{H}$ I alone cannot shift our data points high enough for a perfect match. This is consistent with the fact that we are examining purely atomic sightlines, and likely happens because we are sampling comparatively low number densities $\left(n_{\mathrm{H}} \lesssim 10-100 \mathrm{~cm}^{-3}\right.$; a mixture of WNM and CNM), whereas the sample in PLC2014a includes molecular gas in the $N_{\mathrm{H}}$ bins, presumably with a higher $\kappa_{353}$. However, in diffuse regions with $N_{\mathrm{H}}<5 \times$ $10^{20} \mathrm{~cm}^{-2}$, the mean value of $\sigma_{353}$ from our sample is comparable with that from PLC2014a.

\section{4. $\mathrm{E}(\mathrm{B}-\mathrm{V})$ as the More Reliable Proxy for $\mathrm{N}_{H}$ ?}

We have seen that along 34 atomic sightlines $E(B-V)$ shows a tight linear correlation with $N_{\mathrm{H}}$ in the column density range $N_{\mathrm{H}}=(1 \sim 30) \times 10^{20} \mathrm{~cm}^{-2}$. $\tau_{353}$ also shows a good linear relation with $N_{\mathrm{H}}$ but with systematic deviations as described above.

Figure 11 replicates Figure 10 but for $E(B-V)$ rather than $\tau_{353}$. Although the sample used here is small, these figures demonstrate clearly that the ratio $E(B-V) / N_{\mathrm{H}}$ is more stable than $\tau_{353} / N_{\mathrm{H}}$ over the range of column densities and sightlines covered by our analysis. In fact, with $N_{\mathrm{H}}$ corrected for optical depth effects, our data are compatible with a constant value for $E(B-V) / N_{\mathrm{H}}$, up to $N_{\mathrm{H}}=30 \times 10^{20} \mathrm{~cm}^{-2}$. On the other hand, we have observed an increase of $\tau_{353} / N_{\mathrm{H}}$ with $N_{\mathrm{H}}$, which we suggest may be due to an increase of the dust emissivity (an increase of $r$ and/or $\kappa_{353}$ without significantly affecting the dust absorption cross-section). While we are unfortunately unable to follow how these relations evolve at higher $A_{V}$ and in molecular gas, our results nevertheless suggest that the $E(B-V)$ maps of Green et al. (2018) are a more reliable proxy for $N_{\mathrm{H}}$ than the current release of Planck $\tau_{353}$ in low-tomoderate column density regimes.

\section{OH Abundance Ratio $X_{\mathrm{OH}}$}

The rotational lines of $\mathrm{CO}$ are widely used to probe the physical properties of $\mathrm{H}_{2}$ clouds, but in diffuse molecular regimes where $\mathrm{CO}$ is not detectable in emission other species and transitions must be considered as alternative tracers of $\mathrm{H}_{2}$. Among these, the ground-state main lines of $\mathrm{OH}$ are a promising dark gas tracer; they are readily detectable in translucent/diffuse molecular clouds (e.g., Magnani \& Siskind 1990; Barriault et al. 2010), and since $\mathrm{OH}$ is considered to be a precursor molecule necessary for the formation of $\mathrm{CO}$ in diffuse regions (Black \& Dalgarno 1977; Barriault et al. 2010), it is expected to be abundant in low-CO density/abundance regimes.

The utility of $\mathrm{OH}$ as a tracer of CO-dark $\mathrm{H}_{2}$ depends on our ability to constrain the $\mathrm{OH} / \mathrm{H}_{2}$ abundance ratio, $X_{\mathrm{OH}}=$ $N_{\mathrm{OH}} / N_{\mathrm{H}_{2}}$. From an observational perspective, this requires good estimates of both the $\mathrm{OH}$ and $\mathrm{H}_{2}$ column densities, the latter of which often cannot be observed directly. Many efforts (both modeling and observational) have been devoted to deriving $X_{\mathrm{OH}}$ in different environmental conditions, which we summarize below:

1. Astrochemical models by Black \& Dalgarno (1977) found $X_{\mathrm{OH}} \sim 10^{-7}$ for the case of $\zeta$ Ophiuchi cloud.

2. Nineteen comprehensive models of diffuse interstellar clouds with $n_{\mathrm{H}}$ from 250 to $1000 \mathrm{~cm}^{-3}, T_{\mathrm{k}}$ from 20 to $100 \mathrm{~K}$ and $A_{V}$ from 0.62 to $2.12 \mathrm{mag}$ (van Dishoeck \& Black 1986) found $\mathrm{OH} / \mathrm{H}_{2}$ abundances from $1.6 \times 10^{-8}$ to $2.9 \times 10^{-7}$.

3. The $\mathrm{OH}$ abundance with respect to $\mathrm{H}_{2}$ from chemical models of diffuse clouds was found to vary from $7.8 \times 10^{-9}$ to $8.3 \times 10^{-8}$ with $A_{V}=(0.1-1) \mathrm{mag}$, $T_{K}=(50-100) \mathrm{K}$ and $n=(50-1000) \mathrm{cm}^{-3}$ (Viala 1986).

4. Six model calculations (that differ in depletion factors of heavy elements and cosmic-ray ionization rate) by Nercessian et al. (1988) toward molecular gas in front of the star HD 29647 in Taurus found $\mathrm{OH} / \mathrm{H}_{2}$ ratios between $5.3 \times 10^{-8}$ and $2.5 \times 10^{-6}$. 
5. From $\mathrm{OH}$ observations toward high-latitude clouds using the $43 \mathrm{~m}$ NRAO telescope, Magnani et al. (1988) derived $X_{\mathrm{OH}}$ values between $4.8 \times 10^{-7}$ to $4 \times 10^{-6}$ in the range of $A_{V}=(0.4-1.1) \mathrm{mag}$, assuming that $N_{\mathrm{H}_{2}}=9.4 \times$ $10^{20} A_{V}$. However, we note that the excitation temperatures of the $\mathrm{OH}$ main lines were assumed to be equal, $T_{\mathrm{ex}, 1665}=T_{\mathrm{ex}, 1667}$, likely resulting in overestimation of $N_{\mathrm{OH}}$ (see Crutcher 1979; Dawson et al. 2014).

6. Andersson \& Wannier (1993) obtained an OH abundance of $\sim 10^{-7}$ from models of halos around dark molecular clouds.

7. Combining $N_{\mathrm{OH}}$ data from Roueff (1996) and Felenbok \& Roueff (1996) with measurements of $N_{\mathrm{H}_{2}}$ from Savage et al. (1977, using UV absorption), Rachford et al. (2002, using UV absorption) and Joseph et al. (1986, using CO emission), Liszt \& Lucas (2002) find $X_{\mathrm{OH}}=(1.0 \pm 0.2) \times 10^{-7}$ toward diffuse clouds.

8. Weselak et al. (2010) derived $\mathrm{OH}$ abundances of $(1.05 \pm 0.24) \times 10^{-7}$ from absorption-line observations of five translucent sightlines, with molecular hydrogen column densities $N_{\mathrm{H}_{2}}$ measured through UV absorption by (Rachford et al. 2002, 2009).

9. Xu et al. (2016) report that $X_{\mathrm{OH}}$ decreases from $8 \times 10^{-7}$ to $1 \times 10^{-7}$ across a boundary region of the Taurus molecular cloud, over the range $A_{V}=0.4-2.7 \mathrm{mag} . N_{\mathrm{H}_{2}}$ was obtained from an integration of $A_{V}$-based estimates of the $\mathrm{H}_{2}$ volume density (assuming $N_{\mathrm{H}_{2}}=9.4 \times 10^{20} A_{V}$ ).

10. Recently, Rugel et al. (2018) report a median $X_{\mathrm{OH}} \sim 1.3 \times 10^{-7}$ from THOR Survey observations of $\mathrm{OH}$ absorption in the first Milky Way quadrant, with $N_{\mathrm{H}_{2}}$ estimated from ${ }^{13} \mathrm{CO}(1-0)$.

Overall, while model calculations tend to produce some variation in the $\mathrm{OH}$ abundance ratio over different parts of parameter space $\left(8 \times 10^{-9}-4 \times 10^{-6}\right)$, observationally determined measurements of $X_{\mathrm{OH}}$ cluster fairly tightly around $10^{-7}$, with some suggestion that this may decrease for denser sightlines.

In this paper, we determine our own $\mathrm{OH}$ abundances, using the MS data set to provide $N_{\mathrm{OH}}$ and $N_{\mathrm{H}}$; t then employing $\tau_{353}$ and $E$ $(B-V)$ (along with our own conversion factors) to compute molecular hydrogen column densities as $N_{\mathrm{H}_{2}}=\frac{1}{2}\left(N_{\mathrm{H}}-N_{\mathrm{HI}}\right)$. We note that since this dust-based estimate of $N_{\mathrm{H}_{2}}$ cannot be decomposed in velocity space, the $\mathrm{OH}$ abundances are determined in an integrated fashion for each sightline, and not on a component-by-component basis. While $\mathrm{CO}$ was detected along all but one sightline, it was not detected toward all velocity components, meaning that our abundances are generally computed for a mixture of CO-dark and CO-bright $\mathrm{H}_{2}$ (for further details, see Li et al. 2018).

The $\mathrm{OH}$ column densities derived in Section 3 are derived from direct measurements of $T_{\mathrm{ex}}$ and $\tau$. This means that they should be accurate compared to methods that rely on assumptions about these variables (see, e.g., Crutcher 1979; Dawson et al. 2014). In computing $N_{\mathrm{H}}$, we assume that the linear correlations (deduced from $\tau_{353}, E(B-V)$ and $N_{\mathrm{H} \text { I }}$ toward 34 atomic sightlines) still hold in molecular regions. In this manner, estimates of the $\mathrm{OH} / \mathrm{H}_{2}$ abundance ratio can be obtained within a range of visual extinction $A_{V}=(0.25-4.8)$ mag. We note that, of our $19 \mathrm{OH}$-bright sightlines, 5 produce $N_{\mathrm{H}_{2}}$ that is either negative or consistent with zero to within the measurement uncertainties; these are excluded from the analysis.
Figure 12 shows $N_{\mathrm{OH}}$ and $X_{\mathrm{OH}}$ as functions of $N_{\mathrm{H}_{2}}$. We find that $N_{\mathrm{OH}}$ increases approximately linearly with $N_{\mathrm{H}_{2}}$, and the $\mathrm{OH} / \mathrm{H}_{2}$ abundance ratio is approximately consistent for the two methods, with no evidence of and systematic trends with increasing column density. Differences arise due to the overestimation of $N_{\mathrm{H}}$ derived from $\tau_{353}$ along dense sightlines compared to $N_{\mathrm{H}}$ from $E(B-V)$. As discussed in Section 4, $\sigma_{353}$ varies by up to a factor of 2 in the range of $N_{\mathrm{H}}=(1 \sim 30) \times 10^{20} \mathrm{~cm}^{-2}$, whereas the ratio $\left\langle N_{\mathrm{H}} / E(B-V)\right\rangle$ is quite constant. The mean and standard deviation of the $X_{\mathrm{OH}}$ distribution deduced from $E(B-V)$ is $(0.9 \pm 0.6) \times 10^{-7}$, which is close to the canonical value of $\sim 1 \times 10^{-7}$, and double the $X_{\mathrm{OH}}$ from $\tau_{353},(0.5 \pm 0.3) \times 10^{-7}$. We regard the higher value as more reliable.

\section{Conclusions}

We have combined accurate, opacity-corrected H I column densities from the Arecibo Millennium Survey and 21SPONGE with thermal dust data from the Planck satellite and the new $E(B-V)$ maps of Green et al. (2018). We have also made use of newly published Millennium Survey $\mathrm{OH}$ data and information on CO detections from $\mathrm{Li}$ et al. (2018). In combination, these data sets allow us to select reliable subsamples of purely atomic (or partially molecular) sightlines, and hence assess the impact of HI opacity on the scaling relations commonly used to convert dust data to total proton column density $N_{\mathrm{H}}$. They also allow us to make new measurements of the $\mathrm{OH} / \mathrm{H}_{2}$ abundance ratio, which is essential in interpreting the next generation of $\mathrm{OH}$ data sets. Our key conclusions are as follows:

1. H I opacity effects become important above $N_{\mathrm{H} \mathrm{I}}>5 \times$ $10^{20} \mathrm{~cm}^{-2}$; below this value the optically thin assumption may usually be considered reliable.

2. Along purely atomic sightlines with $N_{\mathrm{H}}=N_{\mathrm{HI}}=$ $(1-30) \times 10^{20} \mathrm{~cm}^{-2}$, the dust opacity, $\sigma_{353}=\tau_{353} / N_{\mathrm{H}}$, is $\sim 40 \%$ higher for moderate-to-high column densities than low (defined as above and below $N_{\mathrm{H}}=5 \times 10^{20} \mathrm{~cm}^{-2}$ ). We have argued that this rise is likely due to the evolution of dust grains in the atomic ISM, although large-scale systematics in the Planck data cannot be definitively ruled out. Failure to account for HI opacity can cause an additional apparent rise of the order of $\sim 20 \%$.

3. For purely atomic sightlines, we measure a $N_{\mathrm{H}} / E(B-V)$ ratio of $(9.4 \pm 1.6) \times 10^{21} \mathrm{~cm}^{-2} \mathrm{mag}^{-1}$. This is consistent with Lenz et al. (2017) and Liszt (2014a), but 60\% higher than the canonical value from Bohlin et al. (1978).

4. Our results suggest that $N_{\mathrm{H}}$ derived from the $E(B-V)$ map of Green et al. (2018) is more reliable than that obtained from the $\tau_{353}$ map of PLC2014a in low-tomoderate column density regimes.

5. We measure the $\mathrm{OH} / \mathrm{H}_{2}$ abundance ratio, $X_{\mathrm{OH}}$, along a sample of 16 molecular sightlines. We find $X_{\mathrm{OH}} \sim 1 \times$ $10^{-7}$, with no evidence of a systematic trend with column density. Since our sightlines include both CO-dark and CO-bright molecular gas components, this suggests that $\mathrm{OH}$ may be used as a reliable proxy for $\mathrm{H}_{2}$ over a broad range of molecular regimes.

J.R.D. is the recipient of an Australian Research Council (ARC) DECRA Fellowship (project number DE170101086). D.L. thanks the supports from the National Key R\&D Program 
of China (2017YFA0402600) and the CAS International Partnership Program (No.114A11KYSB20160008). N.M.-G. acknowledges the support of the ARC through Future Fellowship FT150100024. L.B. acknowledges the support from CONICYT grant PFB06. We are indebted to Professor Mark Wardle for providing us with valuable advice and support. We gratefully acknowledge discussions with Dr. Cormac Purcell and Anita Petzler. Finally, we thank the anonymous referee for comments and criticisms that allowed us to improve the paper.

This research has made use of the NASA/IPAC Infrared Science Archive, which is operated by the Jet Propulsion Laboratory, California Institute of Technology, under contract with the National Aeronautics and Space Administration.

\section{ORCID iDs}

Hiep Nguyen (iD https://orcid.org/0000-0002-2712-4156

J. R. Dawson (iD https://orcid.org/0000-0003-0235-3347

Ningyu Tang (D) https://orcid.org/0000-0002-2169-0472

Di Li (i) https://orcid.org/0000-0003-3010-7661

Claire E. Murray (1D https://orcid.org/0000-0002-7743-8129

N. M. McClure-Griffiths (i) https://orcid.org/0000-00032730-957X

L. Bronfman (iD https://orcid.org/0000-0002-9574-8454

\section{References}

Abdo, A. A., Ackermann, M., Ajello, M., et al. 2010, ApJ, 710, 133 Ackermann, M., Ajello, M., Allafort, A., et al. 2012, ApJ, 755, 22 Ackermann, M., Ajello, M., Baldini, L., et al. 2011, ApJ, 726, 81 Allen, R. J., Hogg, D. E., \& Engelke, P. D. 2015, AJ, 149, 123

Allen, R. J., Ivette Rodríguez, M., Black, J. H., \& Booth, R. S. 2012, AJ, 143, 97

Andersson, B.-G., \& Wannier, P. G. 1993, ApJ, 402, 585

Barriault, L., Joncas, G., Lockman, F. J., \& Martin, P. G. 2010, MNRAS, 407, 2645

Bihr, S., Beuther, H., Ott, J., et al. 2015, A\&A, 580, A112

Black, J. H., \& Dalgarno, A. 1977, ApJS, 34, 405

Blitz, L., Bazell, D., \& Desert, F. X. 1990, ApJL, 352, L13

Boggs, P. T., \& Rogers, J. E. 1990, Contemporary Mathematics, 112, 186

Bohlin, R. C., Savage, B. D., \& Drake, J. F. 1978, ApJ, 224, 132

Bolatto, A. D., Wolfire, M., \& Leroy, A. K. 2013, ARA\&A, 51, 207

Crutcher, R. M. 1979, ApJ, 234, 881

Dame, T. M., Hartmann, D., \& Thaddeus, P. 2001, ApJ, 547, 792

Dawson, J. R., Walsh, A. J., Jones, P. A., et al. 2014, MNRAS, 439, 1596

de Vries, H. W., Heithausen, A., \& Thaddeus, P. 1987, ApJ, 319, 723

Destombes, J. L., Marliere, C., Baudry, A., \& Brillet, J. 1977, A\&A, 60, 55

Dickey, J. M., Kulkarni, S. R., van Gorkom, J. H., \& Heiles, C. E. 1983, ApJS, 53,591

Dickey, J. M., McClure-Griffiths, N. M., Gaensler, B. M., \& Green, A. J. 2003, ApJ, 585, 801

Dickey, J. M., Mebold, U., Stanimirovic, S., \& Staveley-Smith, L. 2000, ApJ, 536,756

Diplas, A., \& Savage, B. D. 1994, ApJ, 427, 274

Douglas, K. A., \& Taylor, A. R. 2007, ApJ, 659, 426

Draine, B. T. 2003, ARA\&A, 41, 241

Draine, B. T., \& Fraisse, A. A. 2009, ApJ, 696, 1

Draine, B. T., \& Li, A. 2007, ApJ, 657, 810

Engelke, P. D., \& Allen, R. J. 2018, ApJ, 858, 57

Felenbok, P., \& Roueff, E. 1996, ApJL, 465, L57

Fukui, Y., Okamoto, R., Kaji, R., et al. 2014, ApJ, 796, 59

Fukui, Y., Torii, K., Onishi, T., et al. 2015, ApJ, 798, 6

Glover, S. C. O., \& Mac Low, M.-M. 2011, MNRAS, 412, 337

Glover, S. C. O., \& Smith, R. J. 2016, MNRAS, 462, 3011

Goodman, A. A., Jones, T. J., Lada, E. A., \& Myers, P. C. 1995, ApJ, 448, 748

Gorenstein, P. 1975, ApJ, 198, 95
Górski, K. M., Hivon, E., Banday, A. J., et al. 2005, ApJ, 622, 759

Green, G. M., Schlafly, E. F., Finkbeiner, D., et al. 2018, MNRAS, 478, 651

Grenier, I. A., Casandjian, J.-M., \& Terrier, R. 2005, Sci, 307, 1292

Hartmann, D., \& Burton, W. B. 1997, Atlas of Galactic Neutral Hydrogen (Cambridge: Cambridge Univ. Press)

Haslam, C. G. T., Salter, C. J., Stoffel, H., \& Wilson, W. E. 1982, A\&AS 47, 1

Heiles, C., Perillat, P., Nolan, M., et al. 2001, PASP, 113, 1247

Heiles, C., Reach, W. T., \& Koo, B.-C. 1988, ApJ, 332, 313

Heiles, C., \& Troland, T. H. 2003a, ApJS, 145, 329

Heiles, C., \& Troland, T. H. 2003b, ApJ, 586, 1067

HI4PI Collaboration, Ben Bekhti, N., Flöer, L., et al. 2016, A\&A, 594, A116 Jones, A. P., Fanciullo, L., Köhler, M., et al. 2013, A\&A, 558, A62

Joseph, C. L., Snow, T. P., Jr., Seab, C. G., \& Crutcher, R. M. 1986, ApJ, 309, 771

Kalberla, P. M. W., Burton, W. B., Hartmann, D., et al. 2005, A\&A, 440, 775

Langer, W. D., Velusamy, T., Pineda, J. L., Willacy, K., \& Goldsmith, P. F. 2014, A\&A, 561, A122

Lee, M.-Y., Stanimirović, S., Murray, C. E., Heiles, C., \& Miller, J. 2015, ApJ, 809,56

Lenz, D., Hensley, B. S., \& Doré, O. 2017, ApJ, 846, 38

Li, D., Tang, N., Nguyen, H., et al. 2018, ApJS, 235, 1

Liszt, H. 2014a, ApJ, 783, 17

Liszt, H. 2014b, ApJ, 780, 10

Liszt, H., \& Lucas, R. 1996, A\&A, 314, 917

Liszt, H., \& Lucas, R. 2002, A\&A, 391, 693

Magnani, L., Blitz, L., \& Wouterloot, J. G. A. 1988, ApJ, 326, 909

Magnani, L., \& Siskind, L. 1990, ApJ, 359, 355

Martin, P. G., Roy, A., Bontemps, S., et al. 2012, ApJ, 751, 28

Meisner, A. M., \& Finkbeiner, D. P. 2015, ApJ, 798, 88

Murray, C. E., Stanimirović, S., Goss, W. M., et al. 2015, ApJ, 804, 89

Nercessian, E., Benayoun, J. J., \& Viala, Y. P. 1988, A\&A, 195, 245

Okamoto, R., Yamamoto, H., Tachihara, K., et al. 2017, ApJ, 838, 132

Paradis, D., Dobashi, K., Shimoikura, T., et al. 2012, A\&A, 543, A103

Peek, J. E. G., \& Graves, G. J. 2010, ApJ, 719, 415

Pineda, J. L., Langer, W. D., Velusamy, T., \& Goldsmith, P. F. 2013, A\&A, 554, A103

Planck Collaboration, Abergel, A., Ade, P. A. R., et al. 2014a, A\&A, 571, A11

Planck Collaboration, Abergel, A., Ade, P. A. R., et al. 2014b, A\&A, 566, A55

Planck Collaboration, Ade, P. A. R., Aghanim, N., et al. 2011, A\&A, 536, A19

Planck Collaboration, Fermi Collaboration, Ade, P. A. R., et al. 2015, A\&A, 582, A31

Rachford, B. L., Snow, T. P., Destree, J. D., et al. 2009, ApJS, 180, 125

Rachford, B. L., Snow, T. P., Tumlinson, J., et al. 2002, ApJ, 577, 221

Reach, W. T., Heiles, C., \& Bernard, J.-P. 2015, ApJ, 811, 118

Reach, W. T., Koo, B.-C., \& Heiles, C. 1994, ApJ, 429, 672

Reina, C., \& Tarenghi, M. 1973, A\&A, 26, 257

Remy, Q., Grenier, I. A., Marshall, D. J., \& Casandjian, J. M. 2017, A\&A, 601, A78

Roueff, E. 1996, MNRAS, 279, L37

Rugel, M. R., Beuther, H., Bihr, S., et al. 2018, A\&A, in press (arXiv:1803. 04794)

Savage, B. D., Bohlin, R. C., Drake, J. F., \& Budich, W. 1977, ApJ, 216, 291

Savage, B. D., \& Jenkins, E. B. 1972, ApJ, 172, 491

Schlafly, E. F., Green, G., Finkbeiner, D. P., et al. 2014, ApJ, 786, 29

Schlegel, D. J., Finkbeiner, D. P., \& Davis, M. 1998, ApJ, 500, 525

Schultz, G. V., \& Wiemer, W. 1975, A\&A, 43, 133

Shull, J. M., \& van Steenberg, M. E. 1985, ApJ, 294, 599

Tang, N., Li, D., Heiles, C., et al. 2016, A\&A, 593, A42

Tielens, A. G. G. M., \& Hollenbach, D. 1985a, ApJ, 291, 747

Tielens, A. G. G. M., \& Hollenbach, D. 1985b, ApJ, 291, 722

van Dishoeck, E. F., \& Black, J. H. 1986, ApJS, 62, 109

van Dishoeck, E. F., \& Black, J. H. 1988, ApJ, 334, 771

Viala, Y. P. 1986, A\&AS, 64, 391

Wannier, P. G., Andersson, B.-G., Federman, S. R., et al. 1993, ApJ, 407, 163

Weselak, T., Galazutdinov, G. A., Beletsky, Y., \& Krełowski, J. 2010 MNRAS, 402, 1991

Weselak, T., \& Krełowski, J. 2014, arXiv:1410.3026

Wolfire, M. G., Hollenbach, D., \& McKee, C. F. 2010, ApJ, 716, 1191

$\mathrm{Xu}$, D., Li, D., Yue, N., \& Goldsmith, P. F. 2016, ApJ, 819, 22

Ysard, N., Köhler, M., Jones, A., et al. 2015, A\&A, 577, A110 